This article has been accepted for publication in Monthly Notices of the Royal Astronomical Society (C): 2017 The Authors. Published by Oxford University Press on behalf of the Royal Astronomical Society. All rights reserved. 


\title{
Paving the way for the JWST: witnessing globular cluster formation at $z>3$
}

\author{
E. Vanzella, ${ }^{1 \star}$ F. Calura, ${ }^{1 \star}$ M. Meneghetti, ${ }^{1}$ A. Mercurio, ${ }^{2}$ M. Castellano, ${ }^{3}$ \\ G. B. Caminha, ${ }^{4}$ I. Balestra, ${ }^{5}$ P. Rosati, ${ }^{4}$ P. Tozzi,${ }^{6}$ S. De Barros, ${ }^{7}$ A. Grazian, ${ }^{3}$ \\ A. D'Ercole, ${ }^{1}$ L. Ciotti, ${ }^{8}$ K. Caputi, ${ }^{9}$ C. Grillo, ${ }^{10,11}$ E. Merlin, ${ }^{3}$ L. Pentericci, ${ }^{3}$ \\ A. Fontana, ${ }^{3}$ S. Cristiani ${ }^{12}$ and D. Coe $^{13}$ \\ ${ }^{1}$ INAF - Osservatorio Astronomico di Bologna, via Ranzani 1, I-40127 Bologna, Italy \\ ${ }^{2}$ INAF - Osservatorio Astronomico di Capodimonte, Via Moiariello 16, I-80131 Napoli, Italy \\ ${ }^{3}$ INAF - Osservatorio Astronomico di Roma, Via Frascati 33, I-00078 Monte Porzio Catone (RM), Italy \\ ${ }^{4}$ Dipartimento di Fisica e Scienze della Terra, Università degli Studi di Ferrara, via Saragat 1, I-44122 Ferrara, Italy \\ ${ }^{5}$ University Observatory Munich, Scheinerstrasse 1, D-81679 Munich, Germany \\ ${ }^{6}$ INAF - Osservatorio Astrofisico di Arcetri, Largo E. Fermi, I-50125 Firenze, Italy \\ ${ }^{7}$ Observatoire de Genève, Université de Genève, 51 Ch. des Maillettes, CH-1290 Versoix, Switzerland \\ ${ }^{8}$ Department of Physics and Astronomy, University of Bologna, via Ranzani 1, I-40127 Bologna, Italy \\ ${ }^{9}$ Kapteyn Astronomical Institute, University of Groningen, Postbus 800, NL-9700 AV Groningen, the Netherlands \\ ${ }^{10}$ Dipartimento di Fisica, Università degli Studi di Milano, via Celoria 16, I-20133 Milano, Italy \\ ${ }^{11}$ Dark Cosmology Centre, Niels Bohr Institute, University of Copenhagen, Juliane Maries Vej 30, DK-2100 Copenhagen, Denmark \\ ${ }^{12}$ INAF - Osservatorio Astronomico di Trieste, via G. B. Tiepolo 11, I-34143 Trieste, Italy \\ ${ }^{13}$ Space Telescope Science Institute, 3700 San Martin Drive, Baltimore, MD 21218, USA
}

Accepted 2017 February 7. Received 2017 February 3; in original form 2016 December 8

\begin{abstract}
We report on five compact, extremely young $(<10 \mathrm{Myr})$ and blue $\left(\beta_{\mathrm{UV}}<-2.5, F_{\lambda}=\lambda^{\beta}\right)$ objects observed with VLT/Multi Unit Spectroscopic Explorer at redshifts 3.1169 and 3.235, in addition to three objects at $z=6.145$. These sources are strongly magnified (3-40 times) by the Hubble Frontier Field galaxy clusters MACS J0416 and AS1063. Their delensed halflight radii $\left(R_{\mathrm{e}}\right)$ are between 16 and $140 \mathrm{pc}$, the stellar masses are $\simeq 1-20 \times 10^{6} \mathrm{M} \odot$, the magnitudes are $m_{\mathrm{UV}}=28.8-31.4\left(-17<M_{\mathrm{UV}}<-15\right)$ and specific star formation rates can be as large as $\sim 800 \mathrm{Gyr}^{-1}$. Remarkably, the inferred physical properties of two objects are similar to those expected in some globular cluster formation scenarios, representing the best candidate proto-GCs discovered so far. Rest-frame optical high-dispersion spectroscopy of one of them at $z=3.1169$ yields a velocity dispersion $\sigma_{v} \simeq 20 \mathrm{~km} \mathrm{~s}^{-1}$, implying a dynamical mass dominated by the stellar mass. Another object at $z=6.145$, with delensed $M_{\mathrm{UV}} \simeq-15.3$ ( $m_{\mathrm{UV}} \simeq 31.4$ ), shows a stellar mass and a star formation rate surface density consistent with the values expected from popular GC formation scenarios. An additional star-forming region at $z=6.145$, with delensed $m_{\mathrm{UV}} \simeq 32$, a stellar mass of $0.5 \times 10^{6} \mathrm{M}_{\odot}$ and a star formation rate of $0.06 \mathrm{M}_{\odot} \mathrm{yr}^{-1}$ is also identified. These objects currently represent the faintest spectroscopically confirmed star-forming systems at $z>3$, elusive even in the deepest blank fields. We discuss how proto-GCs might contribute to the ionization budget of the Universe and augment Ly $\alpha$ visibility during reionization. This work underlines the crucial role of JWST in characterizing the rest-frame optical and near-infrared properties of such low-luminosity high- $z$ objects.
\end{abstract}

Key words: gravitational lensing: strong - galaxies: high redshift-galaxies: star formation.

\section{INTRODUCTION}

Globular clusters (GCs) host the most ancient stars in the Universe. Despite being among the most studied stellar systems and despite

^E-mail: eros.vanzella@oabo.inaf.it (EV); fcalura@oabo.inaf.it (FC) the existence of a large variety of models for their formation, the initial conditions and the physical processes driving their growth and characterizing their earliest evolutionary phases are yet to be understood. Nowadays, it has become clear that a GC can no longer be regarded as a simple stellar population, that is, an assembly of coeval stars sharing identical chemical composition. Over the last decade, substantial evidence has been gathered in favour of the 
presence of multiple stellar populations within GCs (Gratton, Sneden \& Carretta 2004; Piotto et al. 2007; D'Antona \& Caloi 2008).

On the theoretical side, much effort has been devoted to the development of new models for the formation and evolution of GCs. In order to take into account the existence of multiple stellar populations, most popular models for $\mathrm{GC}$ formation consider a scenario in which a second generation (SG) of stars forms from the gas ejected by either first generation (FG) asymptotic giant branch (AGB) stars (Cottrell \& Da Costa 1981; D'Antona \& Caloi 2004; D'Ercole et al. 2008) or FG fast-rotating massive stars (FRMS; Prantzos \& Charbonnel 2006; Decressin et al. 2007), as well as massive interacting binaries (de Mink et al. 2009), or even supermassive $\left(M>10^{4} \mathrm{M}_{\odot}\right)$ stars (Denissenkov \& Hartwick 2014).

The predominance of SG stars observed today in most GCs implies a problem that is common to all the scenarios invoking a standard stellar initial mass function (IMF) for FG stars, that is, the so-called mass-budget problem. In fact, for a Salpeter (1955) or a Kroupa (2001) IMF, the gas shed by the massive stars during the H-burning phase, or the gas contained in the envelopes of massive AGBs (D'Antona \& Caloi 2004; D'Ercole et al. 2008; Renzini et al. 2015), is in general too scarce to form a large SG population. One can solve this problem by postulating that the GC precursors are more massive (by factors between 5 and 20) than the objects we observe today (e.g. D'Ercole et al. 2008; Renzini et al. 2015), or alternatively, by invoking a highly non-standard IMF for the FG, particularly rich in massive stars (D'Antona \& Caloi 2004; Downing $\&$ Sills 2007). The latter scenario, however, faces some serious difficulties when considering the maximum amount of helium that can be produced by an FG of supermassive stars (Renzini et al. 2015). A scenario in which proto-GCs are more massive than today's GCs opens the interesting possibility to detect and spatially resolve their stellar emission.

The main scenarios proposed so far to explain multipopulation GCs postulate the formation of FG stars at $z>2$ (e.g. Kruijssen 2015; D'Ercole, D'Antona \& Vesperini 2016). The formation of GCs has also been studied in the context of cosmological models, which predict their birth within high-density regions of galactic discs at $z>2$ (e.g. Kravtsov \& Gnedin 2005), or envisage that FG stars are formed in an intense burst in the centre of a minihalo during major merger events (Trenti, Padoan \& Jimenez 2015), or consider their formation at the centre of primordial dwarf galaxies (Ricotti, Parry \& Gnedin 2016).

The direct observation of the formation of FG stars within highredshift proto-GCs would provide invaluable clues on how to disentangle such a range of GC formation scenarios.

On the observational side, over the last years many studies have been carried out to derive the faint-end slope of the ultraviolet galaxy luminosity function, its faint-end cut-off $M_{\text {lim }}$ and the Lyman-continuum (LyC) photon production efficiency (e.g. Bouwens et al. 2016a). A major uncertainty in the interpretation of these observations is represented by the observed fraction of ionizing radiation that escapes from galaxies and reaches the intergalactic and circumgalactic media (see e.g. Vanzella et al. 2012b; Siana et al. 2015; Grazian et al. 2016). Irrespective of the nature of the contributors, the general consensus is that the faintest objects represent the main producers of the ionizing background at high redshift (Ferrara \& Loeb 2013; Kimm \& Cen 2014; Wise et al. 2014; Bouwens et al. 2015). It is noteworthy that proto-GCs may also represent good candidates for the sources of radiation that reionized the Universe by $z \sim 6$ (e.g. Ricotti 2002; Schaerer \& Charbonnel 2011; Ricotti et al. 2016).
The detection of extremely faint galaxies at high redshift has been reported in the literature, with magnitudes as faint as $M_{\mathrm{UV}} \simeq-14$ both at redshifts 2-3 (e.g. Alavi et al. 2014, 2016) and 6-8 (e.g. Vanzella et al. 2014; Atek et al. 2015; Bouwens et al. 2015, 2016c; Livermore, Finkelstein \& Lotz 2017). These systems are characterized by very low stellar masses, of the order of a few $10^{6}$ solar masses (e.g. Karman et al. 2016). A still open question concerns the nature of the objects belonging to this luminosity domain: are we dealing with dwarf galaxies (e.g. Finlator et al. 2017), H II galaxies (e.g. Terlevich et al. 2016), super star clusters, or extremely compact star clusters or clumps? (i.e. with sizes of the order of a few tens of parsec; Ellis et al. 2001; Kawamata et al. 2015; Livermore et al. 2015; Bouwens et al. 2016b; Vanzella et al. 2016a)? In order to answer this question, we need to derive a few basic physical quantities such as the stellar mass and the star formation rate (SFR), as well as the size of these systems.

Currently, deep and gravitationally lensed fields observed with the Hubble Space Telescope (HST) offer an opportunity to measure such physical properties, even for extremely faint sources. Much progress has recently being driven by deep observations of massive galaxy clusters, carried out in the context of large HST programmes, particularly the Hubble Frontier Fields (HFF) survey (Koekemoer et al. 2014; Lotz et al. 2014, 2016). Exploiting gravitational lensing, cluster cores are used as cosmic telescopes to look deeply into the distant Universe. High-precision lens models can be built using a large number of multiply lensed sources spanning a large redshift range, which however need to be spectroscopically identified with ground-based or HST grism observations (Treu et al. 2015). In particular, in combination with the very efficient integral field spectrograph Multi Unit Spectroscopic Explorer (MUSE) on the VLT (Bacon et al. 2010), ${ }^{1}$ the identification and characterization of Ly $\alpha$ emitting galaxies near the flux limit of the Hubble imaging data, in lensed and blank fields, has become possible (e.g. Caminha et al. 2016c; Karman et al. 2016; Vanzella et al. 2016a).

In this work, we study five systems detected behind two HFF at redshifts 3.1169 and 3.235 and three at 6.145 extracted from deep MUSE observations of Karman et al. (2016), Vanzella et al. (2016a) and Caminha et al. (2016c). Specifically, we provide novel estimates for the size, dynamical mass and spectral energy distribution (SED) fitting using the full-depth HFF photometry and near-infrared spectroscopy for the object at $z=3.1169$ behind the galaxy cluster AS1063 (named ID11, also discussed in Vanzella et al. 2016a and Karman et al. 2016). The redshifts of the remaining four objects have been presented in Caminha et al. (2016c) and used to constrain the lens model of MACS J0416. Here, we focus on their physical properties. We also explore the possibility that some of these sources may represent proto-GC caught during the formation of their stellar FG. The lens models of the two galaxy clusters discussed in this work have been constrained using dozens of multiple systems spectroscopically confirmed at $3<z<6.5$ with MUSE (Caminha et al. 2016a,c).

The paper is structured as follows: in Section 2, the strongly magnified systems are presented together with the lens models and the inferred sizes. Section 3 describes the photometry and the SED fitting used to derive the physical quantities. We discuss the results in Section 4 and conclude in Section 5.

In this work, we assume a flat cosmology with $\Omega_{\mathrm{M}}=0.3, \Omega_{\Lambda}=$ 0.7 and $H_{0}=70 \mathrm{~km} \mathrm{~s}^{-1} \mathrm{Mpc}^{-1}$, corresponding to 7650, 7560 and

\footnotetext{
${ }^{1}$ www.eso.org/sci/facilities/develop/instruments/muse.html
} 


\section{MACS J0416 System (2) $\quad z=6.145$}
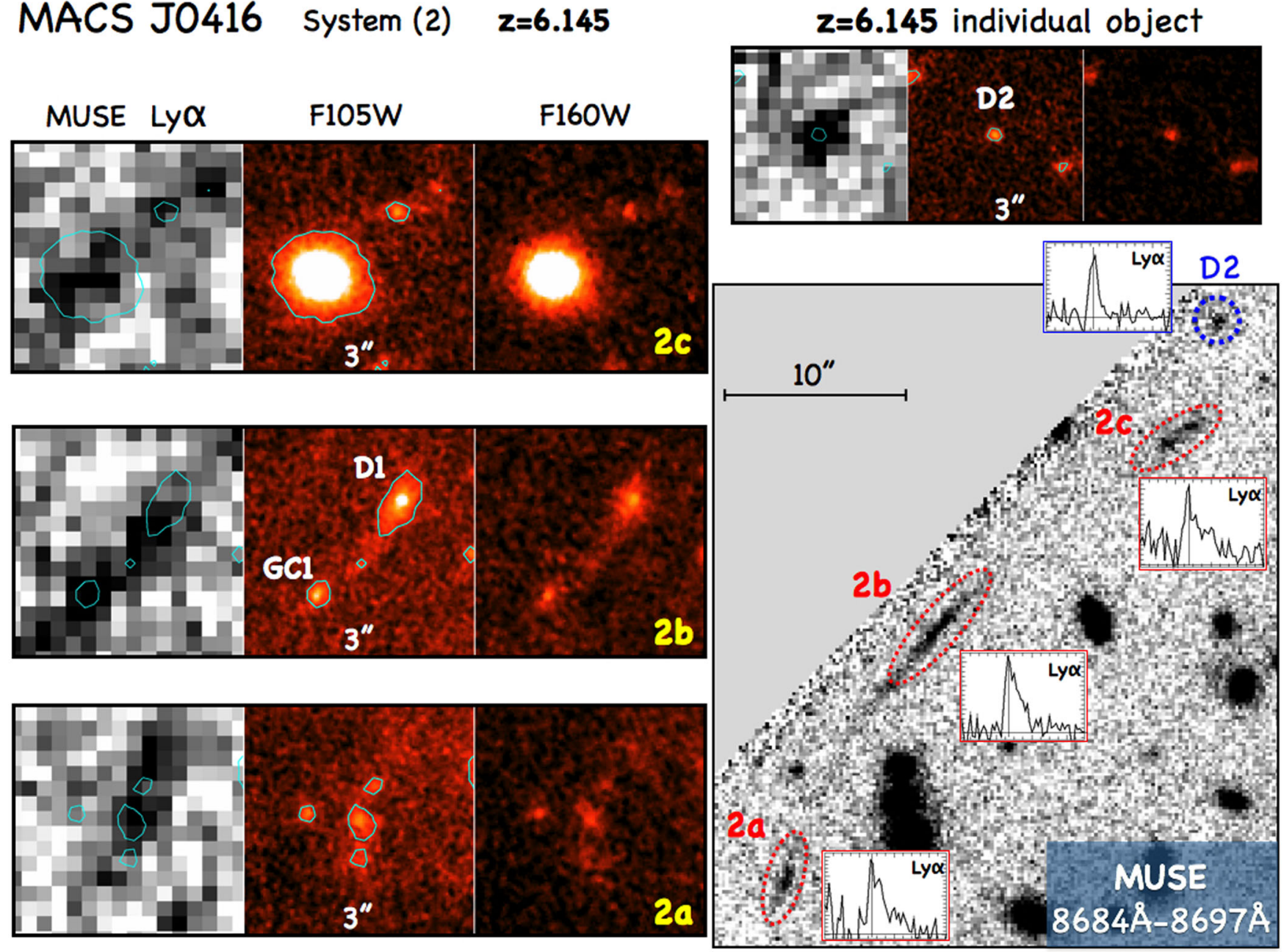

Figure 1. The giant Ly $\alpha$ arc in MACS J0416 (45 arcsec wide) formed by the multiple images 2a, 2b and 2c and the second object (D2) at $z=6.145$ are shown in the HST near-infrared bands (stellar continuum) and MUSE data cube (Ly $\alpha)$. The insets show the one-dimensional Ly $\alpha$ profiles.

5560 physical parsec for 1 arcsec separation at redshifts 3.1169 , 3.235 and 6.145 , respectively.

\section{EXTREMELY SMALL SOURCES IN THE HFF}

The spatial investigation of extremely compact stellar systems $(<100 \mathrm{pc})$ at $z>3$ is precluded in field surveys (as also demonstrated by dedicated simulations presented in Appendix A, see also Bouwens et al. 2016b). As an example, an object with a proper halflight radius of $50 \mathrm{pc}$ at $z=3(6)$ corresponds to 7(9) mas in the sky, or $0.30(0.23)$ pixels, assuming 1 pixel $=0.03$ arcsec (typically the spatial scale of drizzled $H S T$ images). Strong gravitational lensing allows us to probe physical sizes as small as $20-60 \mathrm{pc}$ at $z>3$. This is presented in the next sections.

\subsection{The strongly lensed systems}

Over 100 multiple images have been identified behind the HFF galaxy clusters AS1063 and MACS J0416. For the majority of them, the confirmation of their lensing origin has been possible through MUSE observations (Caminha et al. 2016a,c; Karman et al. 2016). In particular, MUSE integral field spectroscopy has revealed secure Ly $\alpha$ emission from widely separated multiple images. We focus on three systems of multiple images confirmed at redshifts 3.1169 ,
3.235 and 6.145. The angular separation of the multiple images in these systems extends to 50, 20 and 45 arcsec, respectively. These systems are selected on the basis of their strong magnification and high signal-to-noise detection in the HST images ( $\mathrm{S} / \mathrm{N}>10-30$ ). This enables a solid characterization of their sizes and a measurement of their physical properties from SED fitting.

Several images appear distorted tangentially by the cluster mass distribution (as shown in Figs 1 and 2), indicating that they are close to the cluster tangential critical lines (see e.g. Kneib \& Natarajan 2011). In this case, the magnification is dominated by the tangential component $\left(\mu_{\mathrm{T}}\right)$, defined as

$\mu_{\mathrm{T}}=(1-\kappa-|\gamma|)^{-1}$,

where $\kappa$ is the dimensionless surface density (or convergence) and $\gamma$ is the shear. Thanks to this magnification factor, the sources investigated here are spatially resolved along the tangential direction. On the contrary, the radial component of the magnification,

$\mu_{\mathrm{R}}=(1-\kappa+|\gamma|)^{-1}$,

is subdominant at the position of the images. The light profile along the radial direction is consistent with the point spread function (PSF), indicating that the sources are radially unresolved (or marginally resolved at most). The total magnification is $\mu_{\mathrm{tot}}=\mu_{\mathrm{R}} \times \mu_{\mathrm{T}}$. The estimated magnifications for the cases 

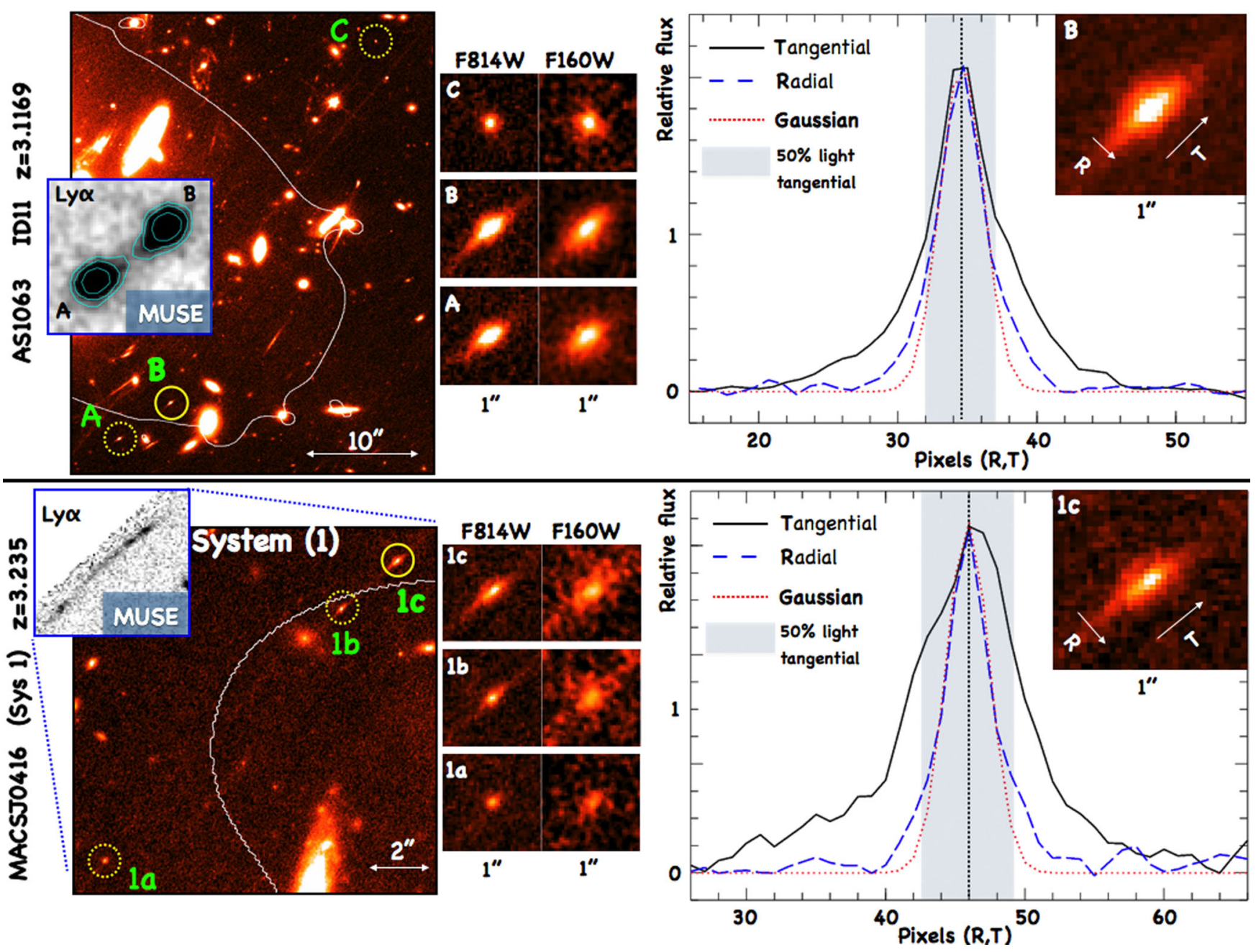

Figure 2. Multiple images and observed light profiles along tangential and radial directions for two sources at redshifts 3.1169 and 3.235 , in AS1063 and MACJ0416, respectively. On the left-hand side, the multiple images of each system are shown in the $F 814 W$ band, including the two-dimensional Ly $\alpha$ emission from VLT/MUSE (insets). The thin white lines represent the critical curves at the redshift of the objects. The zoomed multiple images are also shown in the $F 814 \mathrm{~W}$ and $F 160 \mathrm{~W}$ bands. On the right-hand side, the spatial profiles and images are shown for the most magnified images (image 1c, bottom right, and image $\mathrm{B}$, top right). The object is well resolved along the tangential direction (T), whereas it is not (or barely) resolved along the radial direction (R).

studied in this work are reported in Table 1 and discussed in detail below.

To estimate the half-light radius of the sources $\left(R_{\mathrm{e}}\right)$, we measure the light profiles along the tangential direction. Accounting for the tangential magnification, we derive a delensed value of $R_{\mathrm{e}}$. In this process, we assume that the sources are intrinsically circular and that the observed tangential elongation is only due to magnification.

The circularized effective radius is also calculated (when possible) as $R_{c}=R_{\mathrm{e}} q^{0.5}$, where $q=a / b$ is the axis ratio between the minor and major axes of the source. Such a parameter gives an upper limit when the radial component is not resolved.

In the rest of the work, the effective radii, expressed in parsec, and the physical quantities are always intrinsic (i.e. delensed), whereas radii reported in pixels are by definition observed quantities (1 pixel corresponds to 0.03 arcsec).

\subsection{System ID11 in AS1063 at $\mathrm{z}=3.1169$}

We revisit here the physical size of the object at $z=3.1169$ studied by Vanzella et al. (2016a) and perform SED fitting using the full-depth HFF photometry. Among the sources presented in this work, this is the system with the highest signal-to-noise ratio in the HFF photometry (ranging between 20 and $>50$ for the three multiple images). While the separation between images $\mathrm{A}$ and $\mathrm{C}$ is very large ( $\simeq 50$ arcsec), images $A$ and $B$ are closer, $\lesssim 10$ arcsec, and presumably positioned on opposite sides of the cluster tangential critical line (see the white curve in Fig. 2). Based on the recent analysis by Meneghetti et al. (2016), it is expected that the uncertainty on the magnification estimated from lens models is a steep function of the magnification itself, being $\gtrsim 50$ per cent for $\mu>10$. Although this condition applies to images $\mathrm{A}$ and $\mathrm{B}$, we can obtain a more robust estimate of the magnification of these two images based on the following considerations. In such a 'fold' image configuration, it is expected that the two images have similar magnifications and inverse parity. Indeed, images A and $\mathrm{B}$ have very similar shapes and fluxes $\left(f_{\mathrm{B}} / f_{\mathrm{A}} \simeq 1.1\right)$. Among the three images, the faintest one, $\mathrm{C}$, has the least uncertain magnification factor and flux ratio $f_{\mathrm{B}} / f_{\mathrm{C}}=4.0 \pm 0.05$. These ratios have been inferred by including all the HST/Advanced Camera for Survey (ACS) bands and measuring the average flux ratios among them. In this work, we revise the model of Caminha et al. (2016a) in order to optimally reproduce the observed positions and flux 
Table 1. Intrinsic physical properties and magnifications predicted for the most magnified image of each system (see text for details). Column 1 shows the typical physical quantities expected from the AGB scenario of GC formation (D'Ercole et al. 2008). Columns 3-7 report the inferred properties for the five objects considered in this work: GC1, D1, D2 and Sys_1 identified by Caminha et al. (2016c) and ID11 discussed in Vanzella et al. (2016a). In square brackets, the 68 per cent c.l. is reported (additional constraints from the Ly $\alpha$ line can decrease significantly these uncertainties, see Section 3). Delensed apparent and absolute magnitudes are reported. $f(+) / f(-)$ is the flux ratio between the most and the least magnified images within the same system. (*) Astrodeep IDs (Castellano et al. 2016a; Merlin et al. 2016). (**) These are half-mass radii; they must be reduced by a factor of 1.33 to obtain the two-dimensional half-light radii (Wolf et al. 2010). The age is the elapsed time since the onset of SF.

\begin{tabular}{|c|c|c|c|c|c|c|}
\hline & $\begin{array}{l}\text { Models } \\
\text { Proto-GCs }\end{array}$ & $\begin{array}{c}\mathrm{GC} 1(z=6.145) \\
(*) 2169 \\
04: 16: 11.56 \\
-24: 03: 44.7\end{array}$ & $\begin{array}{c}\mathrm{D} 1(z=6.145) \\
2179 \\
04: 16: 11.48 \\
-24: 03: 43.4\end{array}$ & $\begin{array}{c}\mathrm{D} 2(z=6.145) \\
2411 \\
04: 16: 10.31 \\
-24: 03: 25.8\end{array}$ & $\begin{array}{c}\text { Sys_1c }(z=3.235) \\
2268 \\
04: 16: 11.15 \\
-24: 03: 37.4\end{array}$ & $\begin{array}{c}\text { ID11_B }(z=3.1169) \\
- \\
22: 48: 41.56 \\
-44: 32: 23.9\end{array}$ \\
\hline Stellar mass $\left(10^{6} \mathrm{M}_{\odot}\right)$ & 1,10 & $68_{[21,3273]} \mu_{\text {tot }}^{-1}$ & $380_{[368,585]} \mu_{\text {tot }}^{-1}$ & $16_{[12,1027]} \mu_{\mathrm{tot}}^{-1}$ & $55_{[43,848]} \mu_{\mathrm{tot}}^{-1}$ & $400_{[280,560]} \mu_{\text {tot }}^{-1}$ \\
\hline $\operatorname{SFR}\left(\mathrm{M}_{\odot} \mathrm{yr}^{-1}\right)$ & $0.2,2.0$ & $54_{[1,165]} \mu_{\mathrm{tot}}^{-1}$ & $275_{[131,585]} \mu_{\mathrm{tot}}^{-1}$ & $5_{[0.5,48]} \mu_{\mathrm{tot}}^{-1}$ & $10_{[2,12]} \mu_{\mathrm{tot}}^{-1}$ & $14_{[7,20]} \mu_{\mathrm{tot}}^{-1}$ \\
\hline $\begin{array}{l}\text { Age (Myr) } \\
E(B-V)\end{array}$ & $\stackrel{5}{\sim 0}$ & $1.3_{[1,708]}$ & $1.4_{[1,3]}$ & $3.2[1,710]$ & $5.6_{[4.5,500]}$ & $13_{[2,60]}$ \\
\hline$R_{\mathrm{c}}(\mathrm{UV})(\mathrm{pc})$ & " & $<30$ & $150 \pm 20$ & $<100$ & $<(80-45)$ & $\lesssim 50$ \\
\hline$\Sigma_{\mathrm{SMD}}\left(\mathrm{M}_{\odot} \mathrm{pc}^{-2}\right)$ & $800-1720$ & $1400_{-900}^{+2400}$ & $295_{-80}^{+100}$ & $>85$ & $36-39$ & $1300_{-500}^{+750}$ \\
\hline$\Sigma_{\mathrm{SFR}}\left(\mathrm{M}_{\odot} \mathrm{yr}^{-1} \mathrm{pc}^{-2}\right)$ & $(1.6-3.4) 10^{-4}$ & $2.7 \times 10^{-3}$ & $2.3 \times 10^{-4}$ & $>5.3 \times 10^{-5}$ & $(1.2-1.4) 10^{-5}$ & $1.2 \times 10^{-4}$ \\
\hline$m(1500 \AA)$ & $\simeq 29-32$ & $31.4 \pm 0.2$ & $29.7 \pm 0.2$ & $29.6 \pm 0.3$ & $30.9-32.1$ & $28.8 \pm 0.1$ \\
\hline$M(1500 \AA)$ & $>-17$ & -15.3 & -17.0 & -17.1 & $-15.3,-14.1$ & -17.1 \\
\hline$f(+) / f(-)$ & - & $\simeq 2.5$ & $\simeq 2.5$ & - & $3.7 \pm 0.1$ & $4.00 \pm 0.05$ \\
\hline
\end{tabular}

ratios of the three images of ID11. With this model, we infer a magnification $\mu_{\mathrm{C}}=5.0 \pm 0.2$. The magnification of the counterimages $\mathrm{A}$ and $\mathrm{B}$ is derived from the observed flux ratios between $\mathrm{C}$ and images A and B as in Vanzella et al. (2016a). The resulting total magnifications for images $\mathrm{A}$ and $\mathrm{B}$ are $\mu_{\text {tot }}^{\mathrm{A}}=18.2$ and $\mu_{\text {tot }}^{\mathrm{B}}=20.0$, respectively, with errors smaller than 10 per cent.

As already stated, the images A and B are dominated by the tangential magnification $\left(\mu_{\mathrm{T}}\right)$. Indeed, the radial magnification estimated by the model is quite similar for all three images $\left(\mu_{\mathrm{R}} \simeq\right.$ 1.3), while the tangential magnifications are $\mu_{\mathrm{T}} \simeq 16$ and 15 for images B and A, respectively. As shown in Fig. 2, the light profile along the radial direction is consistent with the PSF, and it is thus unresolved. On the contrary, we can spatially resolve the core and the light profile of the source in the tangential direction.

In order to measure the intrinsic (i.e. unlensed) size of ID11, we use the GALFIT software (Peng et al. 2002, 2010) to fit the sources in both images in the $F 814 W$ band (probing $2000 \AA$ rest frame) and $F 160 \mathrm{~W}$ band (probing $4000 \AA$ rest frame). Then, we use the model magnification to obtain the delensed sizes. The relevant parameters are reported in Table 2 . The tangentially lensed effective radii measured in the $F 814 W(2000 \AA)$ and $F 160 W(4000 \AA)$ bands turn out to be very similar, $R_{\mathrm{e}}(2000 \AA)=3.0 \pm 0.5$ and $R_{\mathrm{e}}(4000 \AA)=4.0 \pm 1.0$ pixels, respectively. This corresponds to $R_{\mathrm{e}}=43 \pm 7 \mathrm{pc}$ and $57 \pm 14 \mathrm{pc}$, respectively $(1 \operatorname{arcsec}=7650 \mathrm{pc}$ at $z=3.1169$ ), after delensing. The delensed circularized effective radius is $R_{\mathrm{c}}=R_{\mathrm{e}} \times q^{0.5} \simeq 50$ pc.

Fig. 2 shows that the light profile is far from a simple Gaussian shape; rather it turns out to follow a Sérsic profile with index $n \simeq 4$ (Fig. 3). In addition, the light profiles are quite symmetric, despite the gradient of the magnification along the tangential direction being large (being close to the critical line). This further supports the intrinsic compactness of the source. In fact, a lensed, more extended object in the source plane would deviate from the symmetric profile generating a boosted tail in the direction of the critical line, towards which the tangential magnification increases rapidly. In our case, the observed stellar continuum follows a symmetric profile instead, showing that differential magnification across the image is negligible. On the contrary, this effect is detected in the two-dimensional Ly $\alpha$ emissions of images A and B. Fig. 2 shows the Ly $\alpha$ emissions averaged over five slices in the MUSE data cube (see also Karman et al. 2015). In this case, the asymmetric shape is observed as two 'asymmetric-lobes' pointing towards the critical line in the middle (see the inset of Fig. 2). This suggests that the Ly $\alpha$ emission arises from a region that is larger than the stellar continuum measured in the $F 814 W$ band, which almost touches the lens caustic on the source plane.

In Appendix A, we describe a set of end-to-end simulations, which validate our method to derive intrinsic radii based mainly on the tangential magnification. Simulations show that the structural properties of the sources are recovered using our procedure and provide further evidence supporting our conclusions on the size and on the brightness profile of ID11.

\subsection{System 1 in MACS J0416 at $\mathrm{z}=\mathbf{3 . 2 3 5}$}

This strongly lensed object is identified as system (1) (Sys_1) in Caminha et al. (2016c). The bottom-left panel of Fig. 2 shows the Ly $\alpha$ emission as a continuous arc-like shape at $z=3.235$, captured in the MUSE data cube, and the three multiple images $1 \mathrm{a}, 1 \mathrm{~b}$ and $1 \mathrm{c}$ generating such a line emission (extending up to $\simeq 17$ arcsec). Also in this case, all the multiple images are well detected in the HST images with $\mathrm{S} / \mathrm{N} \simeq 10-50$ and the geometry of the system is very similar to ID11. We focus on the tangential direction, which allows us to resolve and extract firm constraints on the physical size of the core of the object. In particular, following the same methodology discussed above, we start from the least magnified image 1a and rescale it to the observed flux ratio $f_{1 \mathrm{c}} / f_{1 \mathrm{a}}=3.7 \pm 0.1\left(\simeq \mu_{1 \mathrm{c}} / \mu_{1 \mathrm{a}}\right)$. This ratio was inferred by including all the HST/ACS bands and 
Table 2. Observed morphological parameters derived with GALFIT for the most magnified images. Morphological parameters have been derived in the $F 814 W$ and $F 105 W$ bands for redshifts 3 and 6, respectively, probing $2000 \AA$ and $1500 \AA$. (*) Gaussian fit, object marginally resolved.

\begin{tabular}{lccccc}
\hline Morph. parameters & $\mathrm{GC} 1(z=6.145)$ & $\mathrm{D} 1(z=6.145)$ & $\mathrm{D} 2(z=6.145)$ & Sys_1c( $z=3.235)$ & ID11_B $(z=3.1169)$ \\
& 2169 & 2179 & 2411 & 2268 & - \\
& $04: 16: 11.56$ & $04: 16: 11.48$ & $04: 16: 10.31$ & $04: 16: 11.15$ & $22: 48: 41.56$ \\
& $-24: 03: 44.7$ & $-24: 03: 43.4$ & $-24: 03: 25.8$ & $-24: 03: 37.4$ & $-44: 32: 23.9$ \\
\hline$R_{\mathrm{e}}(\mathrm{pix})$ & $1.7 \pm 0.7$ & $8.3 \pm 0.5$ & $\lesssim 1.5(*)$ & $6.6 \pm 2.1$ & $3.0 \pm 0.5$ \\
$q=b / a$ & $<0.15$ & $0.20 \pm 0.02$ & $0.3 \pm 0.2$ & $<0.15$ & $0.10 \pm 0.02$ \\
$R_{\mathrm{c}}(\mathrm{pix})$ & $<0.70$ & $3.71 \pm 0.23$ & $\lesssim 1.0$ & $<2.5$ & $0.95 \pm 0.17$ \\
PA (deg) & $-39.0 \pm 3.0$ & $-28.5 \pm 0.6$ & $81 \pm 10$ & $-47.0 \pm 2.0$ & $-49.59 \pm 0.5$ \\
$n$ (Sérsic) & $0.5-8$ & $3.0 \pm 0.3$ & 0.5 & $8.0 \pm 2.0$ & $4.0-5.0$ \\
mag & $>27.3$ & $26.1 \pm 0.2$ & $27.8 \pm 0.8$ & $26.9 \pm 0.1$ & $25.3 \pm 0.1$ \\
\hline
\end{tabular}
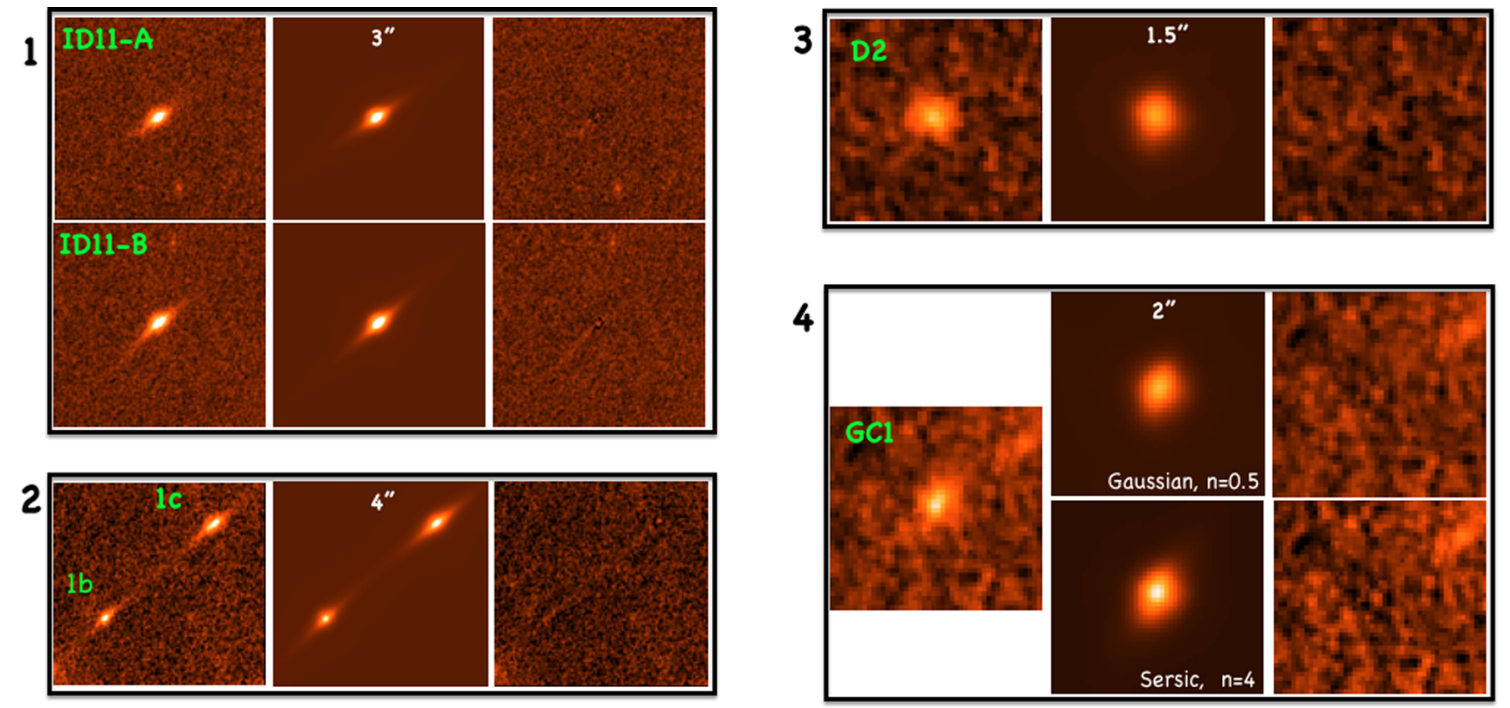

Figure 3. GALFIT fitting of the four compact and most magnified objects in this study: ID11-A and B at $z=3.1169$ (panel 1), the system 1c,b at $z=3.235$ (panel 2), and D2 (panel 3) and GC1 (panel 4) at $z=6.145$. The observed images, GALFIT models and residuals (observed models) are shown from left to right for each system. The morphological parameters are reported in Table 2.

measuring the average flux ratio among them. While the direct estimates of $\mu_{1 \mathrm{~b}}$ and $\mu_{1 \mathrm{c}}$ are affected by large errors, image 1a is the less magnified and the more stable among the others. From the lens model of Caminha et al. (2016c), we derive $\mu_{1 \mathrm{a}}=30 \pm 15$, and from seven different models available from the HFF lens tool calculator ${ }^{2}$ its median value is $\left\langle\mu_{1 \mathrm{a}}\right\rangle \simeq 15$ (all models providing a magnification higher than 10). Therefore, the total $\mu_{1 \mathrm{c}}$, after rescaling by the flux ratio $(\simeq 3.7)$, ranges between 37 and 110 for values of $\mu_{1 \mathrm{a}}$ between 10 and 30 . However, even adopting the lowest $\mu_{1 \mathrm{a}}=10$, the resulting total magnification for image $1 \mathrm{c}$ is $\mu_{1 \mathrm{c}}>37$. Also in this case, the quite elongated shape visible in the $1 \mathrm{c}$ image and the overall Ly $\alpha$ arc suggest that the magnification is mainly tangential. As discussed in the case of ID11, this is evident from the measured $q=b / a=0.10 \pm 0.015$ of image $1 \mathrm{c}$. The predicted tangential magnification for image $1 \mathrm{c}$ is therefore large and spans the range $\mu_{\mathrm{T}}=19-33$, depending on the total magnification assumed (37110) and assuming that the source is circular. Fig. 2 and Table 1 show the result of the GALFIT fitting, which provides a delensed tangential effective radius of $R_{\mathrm{e}}=6.6 \pm 2.1$ pixels. This corresponds to a physical size of 45 (80) pc adopting the tangential magnification $\mu_{\mathrm{T}}$ $=33(19)$. The object is detected also in the $F 160 \mathrm{~W}$ band and shows

\footnotetext{
${ }^{2}$ https://archive.stsci.edu/prepds/frontier/lensmodels/
}

an elongated shape; however, the inferred $R_{\mathrm{e}}$ is strongly affected by the low $\mathrm{S} / \mathrm{N}$ ratio and a tentative estimate ranges between 70 and 150 pc (see inset of Fig. 2, rest-frame $4000 \AA$ ).

\subsection{System 2 in MACS J0416 at $\mathrm{z}=\mathbf{6 . 1 4 5}$}

\subsubsection{The giant arc}

Fig. 1 shows the Ly $\alpha$ emission arising from a giant arc extending for $\sim 45$ arcsec on sky and composed by three multiple images $2 \mathrm{a}$, $2 b$ and $2 c$ (system 2 of Caminha et al. 2016c). The large extension of the arc and the geometry of the multiple images suggest that the source is near the cusp of the lens tangential caustic and that the tangential magnification is again predominant. As expected in a cusp system, image $2 b$ is the most magnified among the three images. Its median magnification among the 11 lens models included in the HFF lens tool calculator is $\mu_{\text {tot }} \simeq 30$. The estimates from all models span the interval $10 \leq \mu_{\text {tot }} \leq 80$. However, these models have been generated without including this system (and many others now available from Caminha et al. 2016c) as a constraint. As shown in fig. 24 of Meneghetti et al. (2016), uncertainties on image magnifications are strongly reduced if nearby multiple images are used as constraints for the lens model. Therefore, we use the HFF models only as an estimate of the systematic uncertainties and we 
adopt the specific modelling presented in Caminha et al. (2016c), in which system 2 has been included and the positions of its multiple images are reproduced. Table 1 reports the total magnifications, together with the tangential and radial components for image $2 b$. While $\mu_{\mathrm{R}}$ is modest and quite stable among the images $(\simeq 1.2-1.4)$ and does not allow us to spatially resolve the emitting sources in the radial direction, the diffuse stellar emission of $2 b$ appears very elongated tangentially, as the Ly $\alpha$ emission detected in the MUSE data cube (Fig. 1).

In the following, we focus on the most magnified of the images, 2b. We identified two distinct (sub)systems in this image, named D1 and GC1, separated by 1.7 arcsec. This angular separation corresponds to a physical delensed separation of $0.6 \mathrm{kpc}$. GC1 is the most compact among the two, though slightly spatially resolved along the tangential direction (see Fig. 1). The two objects are also present in the Astrodeep photometric catalogue with IDs 2179 and 2169, respectively (Castellano et al. 2016a). Their physical properties are reported in Table 1 and discussed in Section 3. The identification of these two sources in the other multiple images of the arc, $2 \mathrm{a}$ and $2 \mathrm{c}$, is difficult, since they are very faint (expected to be $\gtrsim 1.0 \mathrm{mag}$ fainter than $2 \mathrm{~b}$ ) and possibly contaminated by lower redshift cluster members. For example, the expected magnitudes of images $2 \mathrm{a}$ and 2 c of GC1 are $\gtrsim 29.2$, while D1 is brighter and possibly identified, though contaminated by a galaxy cluster member (see Fig. 1).

Given the complex identification of the subsystems GC1 and D1 in the HST images, we use the Ly $\alpha$ fluxes detected in the MUSE data cube as a proxy for the relative ratios among magnifications. We assume that the observed multiple Ly $\alpha$ lines arising from 2a, 2b and $2 \mathrm{c}$ (see Fig. 1) probe the same lensed structure (e.g. the sum of $\mathrm{GC} 1$ and D1). The Ly $\alpha$ flux has been measured on three apertures defined on top of the Ly $\alpha$ emissions (2a, 2b and $2 \mathrm{c}$ ) following the curvature of the arc and calculated collapsing six spectral elements of $1.25 \AA$ each, as the best $\mathrm{S} / \mathrm{N}$ estimate (and corresponding to $\Delta v \simeq$ $300 \mathrm{~km} \mathrm{~s}^{-1}$ at $\left.z=6.145\right)$. The resulting ratios are $\operatorname{Ly} \alpha(2 \mathrm{~b}) / \operatorname{Ly} \alpha(2 \mathrm{a})$ $\simeq \operatorname{Ly} \alpha(2 \mathrm{~b}) / \operatorname{Ly} \alpha(2 \mathrm{c}) \simeq 2.5 \pm 0.7$, and they provide an estimate of the relative magnifications between $2 \mathrm{~b}$ and $2 \mathrm{a}, \mathrm{c}$. The lens model of Caminha et al. (2016c) reproduces the positions and magnitudes of the three multiple images. In particular, the total and tangential magnifications for objects GC1 and D1 calculated for image $2 b$ (i.e. in the most magnified image) are reported in Table 1. Interestingly, the delensed magnitude of GC1 is 31.40 in the $F 105 W$ band (27.88 \pm 0.08 observed; Castellano et al. 2016a), the faintest spectroscopically confirmed object currently known at this redshift, and fainter than any source detected in the current deepest fields (e.g. the Hubble Ultra Deep field, HUDF; Beckwith et al. 2006).

\subsubsection{A faint and dense stellar system at $z=6: G C 1$}

The large tangential magnification allows us to resolve the onedimensional half-light radius. To this aim, we measure the full width at half-maximum of the light profiles in the $F 105 \mathrm{~W}$ band (probing the rest frame $1500 \AA$ ) along the tangential direction, both on the image directly and by using GALFIT modelling. Here, we focus on $\mathrm{GC} 1$, which is the smallest of our objects. It is the faintest object currently confirmed at this redshift with a delensed magnitude of 31.4 (at a $1500 \AA$ rest frame) and with a reasonably high S/N ratio in the photometry, also showing a rest-frame Ly $\alpha$ equivalent width of the order of $100 \AA$ (correcting for the UV slope, see Table 1).

The observed spatial tangential profile of GC1 is shown in Fig. 4, in which 50 per cent of the light is enclosed within $\simeq 5.5-6$ pixels as obtained with GALFIT taking into account the PSF. We explored a large grid of the most relevant parameters, the Sérsic index, effective radius, total magnitude, location of the source, position angle and axis ratio $(q=a / b)$. To accomplish this task, we have followed two different routes: (1) allowing GALFIT to minimize its internal merit function and (2) following the method described by Vanzella et al. (2015), by running GALFIT on a large grid of (fixed) values and monitoring the residuals of the 'observed-model' image, step by step. While the light profile is not reliably constrained (e.g. we obtain a good fit with both Gaussian and Sérsic $n=4$ profiles, see Fig. 3), the size in all cases is relatively well constrained with $R_{\mathrm{e}}$ not greater than 3 pixels (at most). Specifically, the best estimate obtained in the case of a Gaussian ( $n=0.5$ ) profile is $R_{\mathrm{e}}=1.4$ pixels. An estimate of the uncertainties has been obtained by inserting simulated images with sizes and magnitudes similar to those of GC1 (Fig. 4), including also the local noise and background gradients due to the galaxy cluster, and analysed with GALFIT following the same procedure used for GC1. All the parameters are well recovered on average, with a scatter that provides the typical statistical error (the results are reported in Fig. 4). We conclude that the error associated with the observed effective radius of GC1 is of the order of half a pixel in this specific case. Systematic uncertainties, however, could dominate the error budget, in particular the unknown light profile and total magnitude. For example, we allowed the source to be 0.4 mag brighter (27.50) than the measured $F 105 W$ flux $(27.88 \pm 0.09)$ and explored Sérsic profiles up to $n=10$. The effective radii are 0.9 , 1.4 and 2.1 pixels in the case of magnitude 27.50 and $n=8,4$ and 0.5 (Gaussian), respectively, while it is smaller than 1.2 pixels in all the cases with magnitude 27.80 (the observed value). Examples of GALFIT models are shown in Fig. 3. We conclude that a plausible estimate of the radius is $R_{\mathrm{e}}=1.7 \pm 0.7$ pixels (Fig. 4).

At $z=6.145$, with the tangential magnification computed above, $\mu_{\mathrm{T}}=17.5$, the inferred radius is $R_{\mathrm{e}}=16 \pm 7 \mathrm{pc}$. The circularized radius is therefore $R_{\mathrm{c}} \simeq 20 \mathrm{pc}$, adopting $q=0.15$.

Even considering conservative estimates for the radius and magnification (i.e. $R_{\mathrm{e}}=2.4$ pixels and $\mu_{\mathrm{T}}>10$ ), the size still remains remarkably small, $R_{\mathrm{e}}<40 \mathrm{pc}$.

\subsubsection{The system D1 and additional extremely faint SF knots}

The same GALFIT fitting has been performed for D1, the most extended object among those studied in this work and close to GC1, both at $z=6.145$. A clear nucleated star-forming region surrounded by a diffuse emission extending approximately $\simeq 200 \mathrm{pc}$ along the tangential component is evident (Fig. 5). We obtain an effective radius $R_{\mathrm{e}} \simeq 140 \mathrm{pc}$, making this object compatible with a forming dwarf galaxy. A morphological decomposition is beyond the scope of this work, but it is worth noting that the spatial distribution of the nuclear emission is quite symmetric despite the large magnification along the tangential direction (see the $10 \sigma$ contours in Fig. 5). As discussed above, such a symmetric shape implies that the size of the inner part is extremely compact also for D1. The radius of the region enclosed within $10 \sigma$ from the background is $\sim 0.12 \operatorname{arcsec} \simeq$ $50 \mathrm{pc}$, suggesting a dense and very nucleated star formation activity.

Looking carefully at image $2 b$, we identify even fainter features. Fig. 5 shows the identification of an additional knot between D1 and GC1, identified as ID $=22692$ in the Astrodeep catalogue. Despite the strong lensing magnification, its observed magnitude of $F 105 W \simeq 28.5$, implies a delensed $F 105 W \simeq 32$ (i.e. $M_{\mathrm{UV}}=-14.7$, adopting the aforementioned total magnification, 25). From the SED fitting, we derive an intrinsic stellar mass of $M \simeq 0.5-0.6 \times 10^{6} \mathrm{M}_{\odot}$ and an $\mathrm{SFR} \simeq 0.06 \mathrm{M}_{\odot} \mathrm{yr}^{-1}$. Although these measurements are still uncertain, lensed substructures like this one provide a first glimpse into a completely unexplored luminosity, mass domain at these 

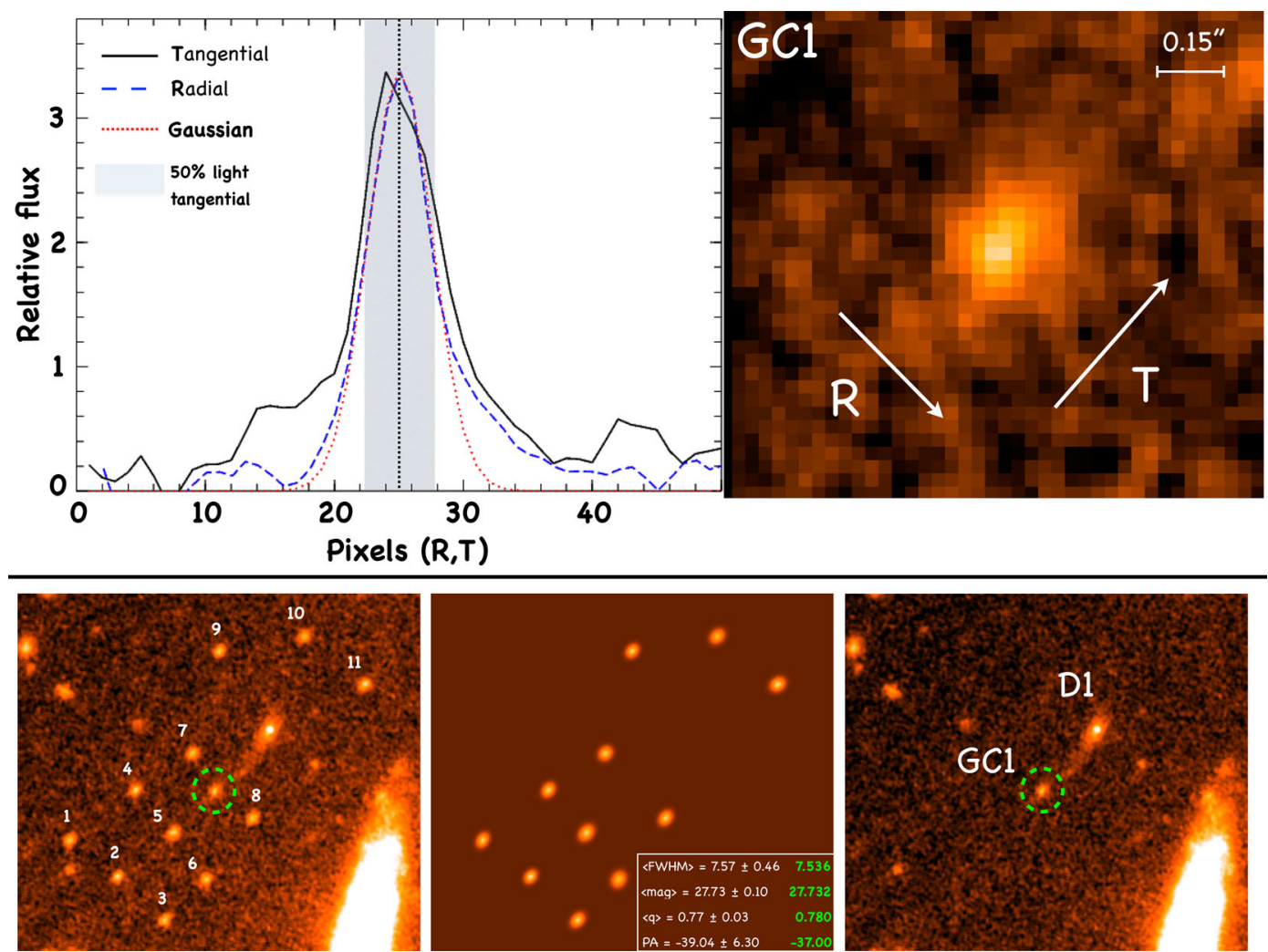

Figure 4. Top left: light profiles of the source $\mathrm{GC} 1$, at $z=6.145$, along the radial (R) and tangential (T) directions. The range including 50 per cent of the light along $\mathrm{T}$ is marked with a grey region, while the profile is consistent with the PSF along R. The radial and tangential directions are shown in the GC1 image to the right, where a slightly elongated shape is evident along T. Bottom: 11 simulated images (1-11) inserted around GC1 (dashed green circle) are shown (left); the GALFIT models and residuals are shown in the middle and right-hand panels, respectively. The inset in the middle panel compares the average and standard deviation of the parameters recovered with GALFIT (in white) with the real input values (in green).

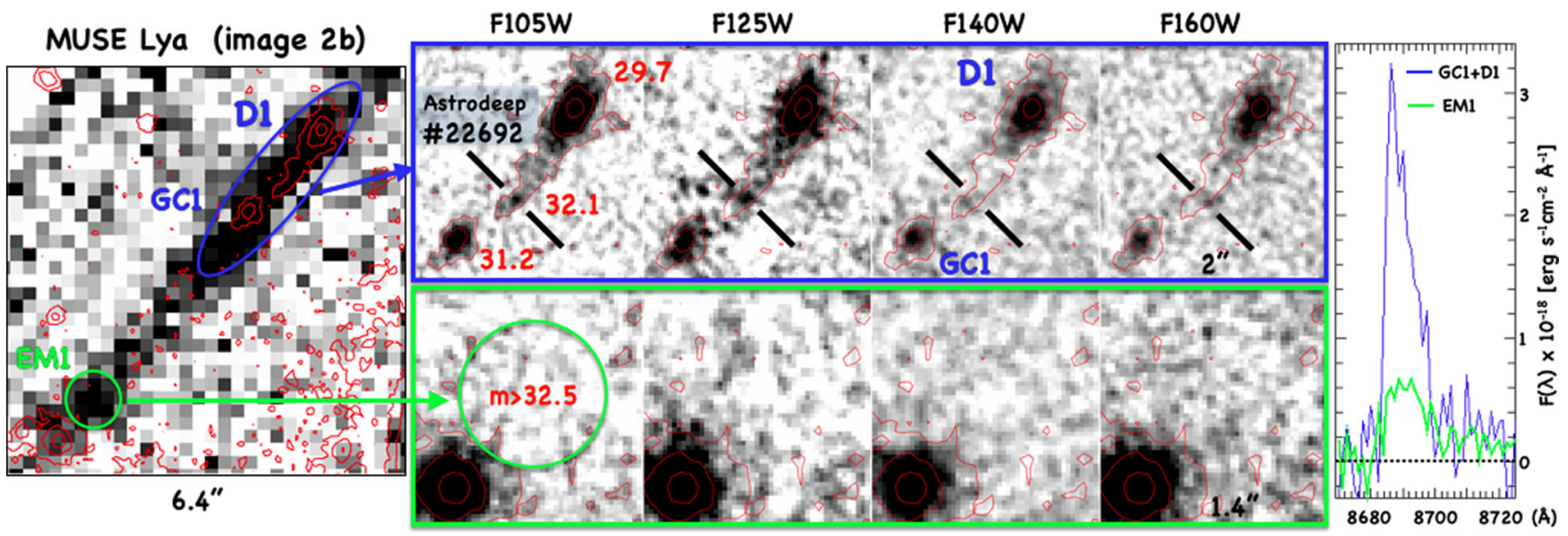

Figure 5. A zoomed view of the Ly $\alpha$ emission (MUSE) of GC1 and D1 (marked with a blue ellipse) at $z=6.145$. The cutouts are shown in the $F 105 W$, $F 125 \mathrm{~W}, F 140 \mathrm{~W}$ and $F 160 \mathrm{~W}$ bands at the HFF depth (magnitude limit 29.4 at $3 \sigma$ within 0.4 arcsec diameter aperture). The red contours show $2 \sigma, 4 \sigma$ and $10 \sigma$ level above the background. D1 shows a clear nucleated core and an elongated emission along the tangential direction, suggesting that it is compact in the inner part with an underlying distorted shape (modulated by the magnification). The $10 \sigma$ contour of the core of D1 appears symmetric despite the large tangential magnification, suggesting that it is significantly compact. A possible stellar stream linking D1 and GC1 is present, traced by the $2 \sigma$ contour. Another knot, marked with ID \#22692 (Astrodeep; Castellano et al. 2016a; Merlin et al. 2016), is detected in the $F 105 W$ and $F 125 W$ bands, and barely in the $F 160 W$ band (top panels), suggesting a steep ultraviolet slope. An Ly $\alpha$ emission feature without any HST counterparts is shown in the bottom panels (EM1, green circle). The Ly $\alpha$ profiles for both GC1 + D1 sources (blue line) and EM1 (green line) are shown to the right. The observed line fluxes are $(3.0 \pm 0.1) \times 10^{-17}$ within a polygonal aperture and $(0.6 \pm 0.2) \times 10^{-17} \mathrm{cgs}$ within a circular aperture of $1.6 \operatorname{arcsec}$ diameter. The delensed magnitudes of each object are shown in red in the $F 105 W$ images. 
redshifts. The GaLFIT fitting does not provide in this case robust results, though this object appears extremely small, at the level of a few parsec. Understanding the nature of forming knots of this kind will require JWST observations. We also note that another Ly $\alpha$ emitting knot is detected in the MUSE data, denoted as 'EM1' in Fig. 5, which does not have any counterpart in the HST images, down to magnitude limits of 29.4 (at $3 \sigma$ level, the HFF depth). If this $\operatorname{Ly} \alpha$ emission is produced by an underlying star formation activity, it would imply that the associated source is fainter than magnitude $\simeq 33$ (i.e. fainter than $M_{\mathrm{UV}}=-13.7$ ) and the resulting rest-frame Ly $\alpha$ equivalent width larger than $300 \AA$. Alternatively, the Ly $\alpha$ emission may arise from fluorescence induced by, e.g. $\mathrm{GC} 1$ and/or D1, suggesting the presence of surrounding neutral gas illuminated by close star-forming activity.

\subsection{The source D2 in MACS J0416 at $\mathrm{z}=6.145$}

From the MUSE data cube, we identified another lensed Ly $\alpha$ emission line of an object (named D2) at the same redshift as system $2(z=6.145)$, which is not part of the same galaxy (see Fig. 1) and has a rest-frame Ly $\alpha$ equivalent width of $140 \AA$. The optical counterpart is well detected in the HFF deep photometry with an $F 105 W$ magnitude of $28.33 \pm 0.09$ (Castellano et al. 2016a; Merlin et al. 2016). The object is located at $\simeq 27 \mathrm{kpc}$ from GC1 in the source plane, and is therefore distinct from system 2 but plausibly part of the same environment of GC1 and D1. The source is well fitted with a two-dimensional Gaussian shape and a Sérsic $n=4$ profile, with errors on the morphological parameters dominated by the relatively low $\mathrm{S} / \mathrm{N}$. In practice, the object is not spatially resolved; therefore, only an upper limit on the effective radius can be obtained. Using GALFIT on a grid of parameters and simulations, as previously done for $\mathrm{GC} 1$, we can exclude an effective radius greater than 1.5 pixels. Therefore, adopting $R_{\mathrm{e}}<1.5$ pixels and $\mu_{\mathrm{tot}} \simeq 3.0 \pm 0.5$ (in this case $\mu_{\mathrm{T}} \simeq \mu_{\mathrm{R}}$ ), we obtain an intrinsic size of $R_{\mathrm{e}} \simeq R_{\mathrm{c}}<150 \mathrm{pc}$.

\section{PHYSICAL PROPERTIES FROM SED FIT TING}

Physical properties of the aforementioned sources have been derived from their SED by means of fits performed with a set of templates from Bruzual \& Charlot (2003), with the addition of nebular continuum and emission lines as described in Castellano et al. (2016a, see also Schaerer \& de Barros 2009). All the objects lensed by the HFF cluster MACS J0416 benefit from the Astrodeep photometric catalogue (Merlin et al. 2016) and the redshift measurements from MUSE observations. SED fitting of the Astrodeep sources in MACS J0416 was presented in Castellano et al. (2016a) on the basis of photometric redshifts: here, we update that analysis by fixing the redshift at the spectroscopic value and allowing also for very young ages (1-10 Myr) that were not previously considered. The source ID11, behind the galaxy cluster AS1063, has already been analysed and described in Vanzella et al. (2016a). Here, we add the deepest near-infrared photometry that was not available at that time. The resulting SED fitting for all our objects is shown in Fig. 6. The relevant parameters are reported in Table 1, that is, stellar masses, ages, $E(B-V)$, SFRs along with their 68 per cent c.l. uncertainties. The observed photometry and SEDs are shown in Fig. 6. It is worth stressing that, despite the extremely faint intrinsic magnitudes involved (between 28.6 and 31.4), the multiband photometry is robust thanks to long HST exposure times in addition to strong lensing magnification. For the whole sample, typical S/N ranges from 10 to $>50$ in the ultraviolet continuum and rest-frame optical wavelengths (for ID11 and Sys_1c). From a linear fit of the observed photometry in the UV rest-frame range (see e.g. Castellano et al. 2012), we measure very blue ultraviolet slopes, ranging between -2.5 and -3.0 , which reflect the typically young ages, very low dust extinction and possibly low metallicities (Castellano et al. 2014). In general, the intrinsic faintness of these sources translates into modest SFRs, of the order of $0.1-1 \mathrm{M}_{\odot}$ and low stellar masses, ranging between 1 and $20 \times 10^{6} \mathrm{M}_{\odot}$. Such low masses have also been measured by Karman et al. (2016) behind AS1063 (including ID11). In the case of GC1, D2 and Sys_1c, solutions at higher masses and old ages ( $>100 \mathrm{Myr}$ ) are allowed by the fit due to larger uncertainties in the $K s$ and IRAC fluxes. This has been verified by applying the photometric errors of GC1 to the D1 object. This affects mainly the optical rest-frame part of the SED, the $K s$ and IRAC bands. The solutions from the SED fitting of D1 show similar degeneracies to those observed for $\mathrm{GC} 1$, confirming that optical rest-frame photometry is critical when inferring the ages and stellar masses. Older and more massive solutions are reported in Fig. 6 (red and magenta lines), in addition to the best-fitting solutions (blue lines). However, the Ly $\alpha$ flux measured from MUSE spectroscopy allows us to strengthen the constraints on physical parameters for the two systems at $z=6.145$. Following Schaerer (2003), and assuming Salpeter IMF and metallicity $Z=0.004$, the observed Ly $\alpha$ luminosity of GC1 and D2 can be converted into a lower limit ${ }^{3}$ of SFR $=4 \mathrm{M}_{\odot} \mathrm{yr}^{-1}$ and $2 \mathrm{M}_{\odot} \mathrm{yr}^{-1}$, respectively. With these additional constraints, no solutions older than 9 Myr are found for $\mathrm{GC} 1$ such that the upper limit on its stellar mass is reduced by a factor of $\sim 20$ from $3.3 \times 10^{9}$ to $1.6 \times 10^{8} \mathrm{M}_{\odot}$ (observed). Similarly, the maximum age allowed for D2 decreases from 710 to $100 \mathrm{Myr}$ with a maximum stellar mass of $2.3 \times 10^{8} \mathrm{M} \odot$ (observed). An example of the aforementioned degeneracy among stellar mass, age and SFR is shown in Fig. 7 for GC1, the smallest of our objects. A main 'cloud' of solutions is visible and spans the intervals 1-10 Myr and $10^{7.3-8.3} \mathrm{M}_{\odot}$ (observed). A second 'cloud' at high masses and older ages is also apparent; however, it is disfavoured if the SFR derived from the Ly $\alpha$ emission is considered ( $\operatorname{SFR}(\operatorname{Ly} \alpha)$ $>4 \mathrm{M}_{\odot} \mathrm{yr}^{-1}$ ). It is also worth stressing that solutions with larger masses would also imply stellar mass densities more than 10 times larger than the best-fitting values reported in Table 1. Therefore, our objects are fully consistent with solutions favouring very young ages, low stellar masses and a low amount of dust. The combination of the sizes and the physical quantities described above allow us to estimate the surface densities of star formation and stellar mass for the objects of our sample. A consequence of the inferred stellar mass and SFR estimates is the very large specific star formation rate $\left(\mathrm{sSFR}=\mathrm{SFR} / \mathrm{M}_{\star}\right)$, a quantity that does not depend on the magnification. Specifically, we derive an SSFR spanning the interval 30-800 $\mathrm{Gyr}^{-1}$, as expected for a young, low-mass object in which a burst of star formation is ongoing (e.g. Karman et al. 2016). In particular, the object $\mathrm{GC} 1$ shows an $\mathrm{sSFR} \simeq 800 \mathrm{Gyr}^{-1}$ and will double its stellar mass in $\lesssim 5 \mathrm{Myr}$.

Overall, it is also worth noting that a significant contribution from the nebular emission lines [O III] $\lambda \lambda 4959,5007, \mathrm{H} \beta$ and $\mathrm{H} \alpha$ is expected in the $K s$ or IRAC bands, at the level of several hundreds or thousands $\AA$ of equivalent width (rest frame). In particular, the nebular contribution in the $K s$ band for ID11 has been well measured with VLT/X-Shooter, allowing us to derive robust estimates of its physical properties from SED fitting (Vanzella et al. 2016a).

\footnotetext{
${ }^{3}$ The Ly $\alpha$ emission can be partially attenuated by a small amount of dust and by the presence of the intergalactic medium.
} 

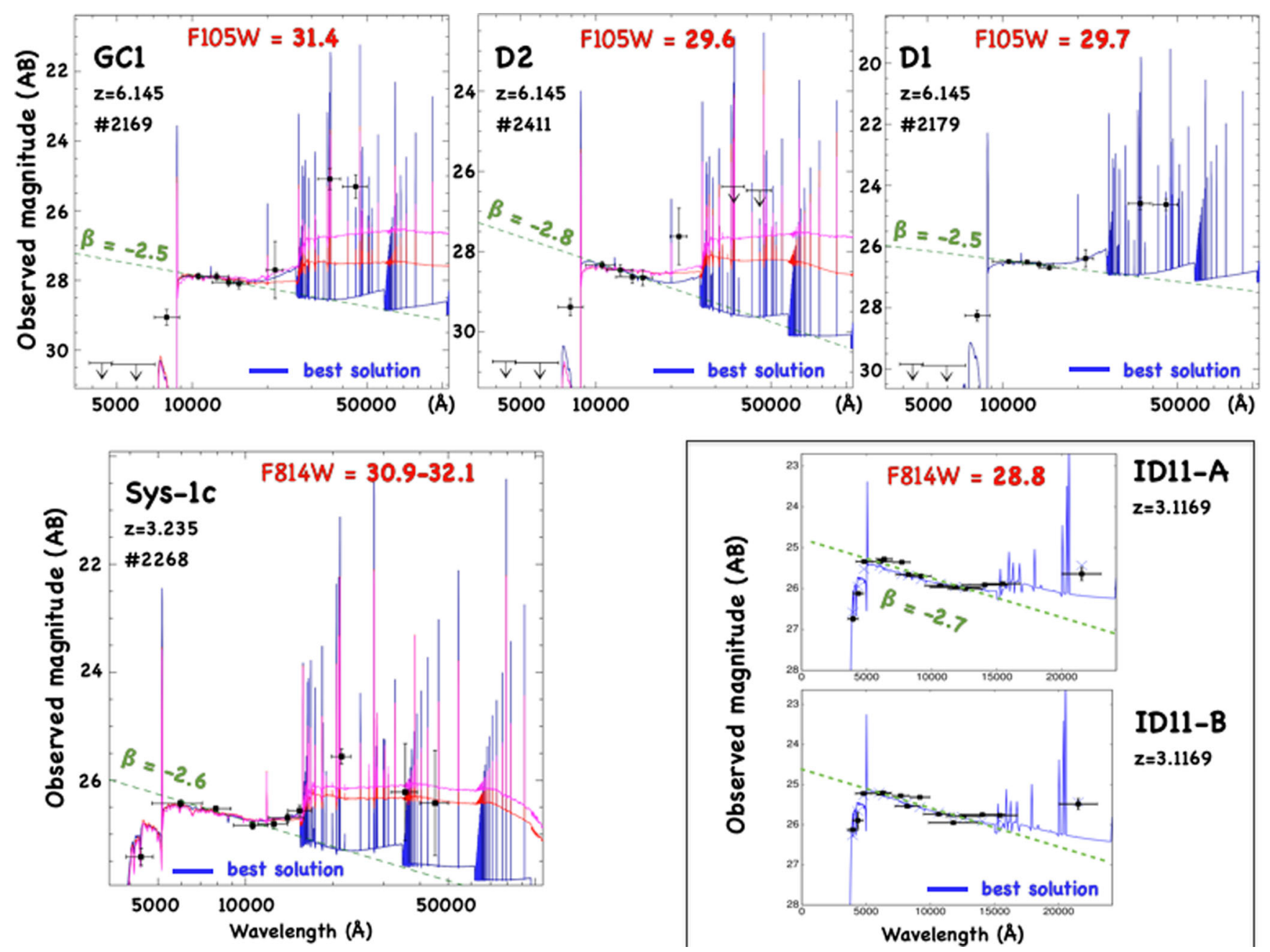

Figure 6. SED fitting for each system in MACS J0416, based on the Astrodeep photometric catalogue (Castellano et al. 2016a; Merlin et al. 2016). The physical quantities derived from the SED fit of ID11 have already been discussed in Vanzella et al. (2016a) and updated here with the deepest HFF photometry. The photometric redshift and the stellar emission including nebular prescription (Castellano et al. 2016a) reproduce well the observed magnitudes. The physical properties are summarized in Table 1. Best-fitting solutions are shown in blue (see Table 1) and when present, old and more massive solutions are reported with red and magenta lines (300 and $700 \mathrm{Myr}$ old, respectively). The ultraviolet slopes $\beta$ are shown along dashed green lines. Delensed $F 105 W$ and $F 814 W$ magnitudes are shown in red in each panel.

Similarly, X-Shooter observations of Sys_1 at $z=3.235$ are under acquisition. For the objects at $z>6$ presented in this work, there is a clear signature of nebular line contribution ([O III] $\lambda \lambda 4959,5007$, $\mathrm{H} \beta$ and $\mathrm{H} \alpha$ ) in the IRAC $3.6 \mu \mathrm{m}$ and $4.5 \mu \mathrm{m}$ channels. The precise intensity and line ratios at $z>6$ will only be measurable in the future when JWST NIRSpec spectroscopy will become available. The rest-frame optical stellar continuum will also be observable with JWST NIRCam.

\section{DISCUSSION}

\subsection{Proto-Globular clusters}

Current scenarios for the formation of multipopulation GCs include a massive FG of stars forming at redshift $z>2$ (e.g. Kruijssen 2015; Renzini et al. 2015). At the present time, little is known about the physical conditions characterizing the stellar FG. From the population of GCs present in the Milky Way (MW), we know that in general and in terms of mass, present-day GCs have subdominant FG populations with respect to the SG, with a very few excep- tions (Bragaglia et al. 2015). Current theoretical multipopulation scenarios for GC formation do not focus much on the events originating the FG. The most popular scenarios, that is, the AGB and the FRMS scenarios, generally start keeping track of the evolution of the system after an FG is already in place, generated by a single, instantaneous burst of star formation at high redshift $(z>2$, e.g. Kruijssen 2015; Renzini et al. 2015). In both scenarios, the SG of stars forms out of the gas shed by FG stars. As the mass return from aging stellar populations is in general too scarce to form a large SG population (assuming a standard IMF; e.g. Calura, Ciotti \& Nipoti 2014; Renzini et al. 2015), in order to account for the present-day mass and predominance of SG stars as observed in GCs, the FG has to be substantially more massive than present-day GCs, by a factor ranging between 5 and 20 (D'Ercole et al. 2008; Renzini et al. 2015). Most of this massive FG has then to be lost via dynamical processes, such as evaporation and tidal interactions. Regarding this aspect, it is noteworthy that a system like CG1 presents hints for a dynamical interaction with the massive companion D1, and for a possible stellar stream between these two objects. This could indicate an already ongoing stellar stripping, as expected in 


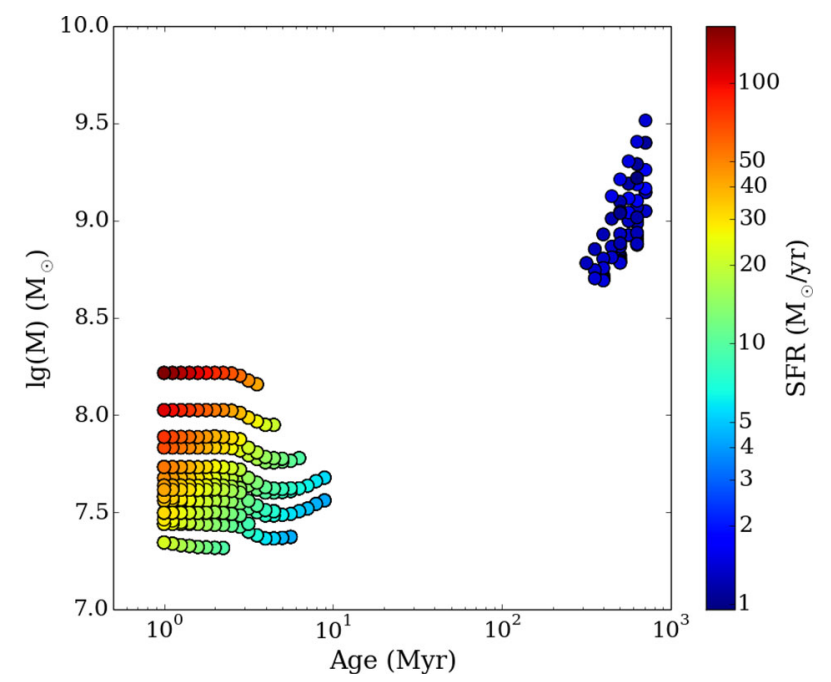

Figure 7. The degeneracy among stellar mass, age and SFR is shown in the case of the SED fitting of GC1. All the solutions within the 68 per cent interval are shown. The reported quantities are derived from the observed photometry, that is, they are not delensed (intrinsic values of the SFR and stellar mass are obtained dividing by $\mu_{\text {tot }}=25$, see Table 1$)$. The SFR is colour coded and indicated on the right of the figure. The minimum SFR inferred from the Ly $\alpha$ emission $\left(>4 \mathrm{M}_{\odot} \mathrm{yr}^{-1}\right)$ favours the solutions at low masses $\left(10^{7.3-8.3} \mathrm{M}_{\odot}\right.$ observed, corresponding to $10^{5.9-6.9} \mathrm{M}_{\odot}$, delensed) and young ages, 1-10 Myr. See the best solutions in Fig. 6.

GC scenarios soon after the formation of the FG (e.g. D'Ercole et al. 2008).

The FRMS scenario (Decressin et al. 2007) assumes an initially highly concentrated cluster with a small half-mass radius, typically of the order of a few pc (Decressin et al. 2010; Krause et al. 2012), with initial total mass densities comparable to the central densities of the most massive clusters observed today $\left(\sim 10^{5} \mathrm{M}_{\odot} \mathrm{pc}^{-3}\right.$; Renzini et al. 2015). The expulsion of the residual gas is assumed to occur on a relatively short time-scale, faster than the crossing time-scale (Decressin et al. 2010), soon after the cease of type II SN explosion as due to a sudden accretion on to the dark remnants (Krause et al. 2012). The loss of the gas causes a sudden change of gravitational potential, which is then able to unbind a large fraction of FG low-mass stars sitting initially in the GC outskirts (Decressin et al. 2010).

The AGB scenario assumes a GC that forms within the disc of a high-redshift galaxy (D'Ercole et al. 2008, 2016). A natural outcome of the AGB scenario is an FG characterized by a flatter density profile than the SG. This is due to the fact that after the explosion of all the $\mathrm{SNe}$ of the FG, the gas shed by the AGB stars originates a cooling flow directed towards the centre of the cluster. As the SG forms out of this gas mixed with some pristine gas (D'Ercole et al. 2016), its distribution will be much more concentrated than the one characterizing the FG (D'Ercole et al. 2008).

A key element that differentiates the AGB and the FRMS scenarios is the size assumed for the stellar FG. The initial size and concentration of the FG is a crucial quantity regulating the subsequent mass-loss experienced by the cluster in the remainder of its history. D'Ercole et al. (2008) study two different models characterized by an FG of mass $10^{7}$ and $10^{6} \mathrm{M}_{\odot}$, which correspond to GCs of present-day masses of $10^{6}$ and $10^{5} \mathrm{M}_{\odot}$, respectively. In both cases, the distribution of the FG follows a King (1962) radial profile, with half-mass radii of $35 \mathrm{pc}$ and $16 \mathrm{pc}$ for initial masses of $10^{7}$ and $10^{6} \mathrm{M}_{\odot}$, respectively. For each model, the truncation radius of the profile corresponds to the tidal radius at a distance of $4 \mathrm{kpc}$ from the Galactic Centre. This results from the assumption that the GC is placed on a circular orbit located at this galactocentric distance and with an external, galactic tidal field modelled as a Keplerian potential generated by a point mass of $M_{\mathrm{g}} \sim 4 \times 10^{10} \mathrm{M}_{\odot}$. These assumptions ensure a significant mass-loss of FG stars due to the external potential. If the energy injected by the FG stellar winds and $\mathrm{SNe}$ is sufficient to expel the $\mathrm{SN}$ ejecta and the residual gas (Calura et al. 2015), the stellar FG can expand beyond its tidal limit in response to this substantial gas loss and be prone to efficient stellar mass-loss due to the external field (D'Ercole et al. 2008). Clearly, the efficiency of this mechanism is sensitive to the parameters regulating the initial FG distribution, as more concentrated stellar distributions will give place to smaller amounts of mass lost via tidal stripping (e.g. Vesperini \& Heggie 1997).

Besides supporting a preferential loss of FG stars, the natural prediction of the AGB scenario of a more concentrated SG is also in agreement with observations of GCs, showing that red stars (generally identified with SG) are always more centrally concentrated than blue stars (generally identified with FG stars; Lardo et al. 2011). These aspects outline another key difference between the AGB and FRMS scenario, that is, that in order to explain the different proportions and radial distributions of FG and SG stars, the latter has to postulate that the SG was formed close to the massive stars in a mass-segregated star cluster (Decressin et al. 2010).

The ab initio study of GC formation of Nakasato, Mori \& Nomoto (2000) starts from a metal-free proto-GC cloud of a few $\sim 100 \mathrm{pc}$, in which a first population of $10^{2} \mathrm{M}_{\odot}$ of metal-free stars originate, enriching the cloud with heavy elements and whose self-generated emission of photodissociative photons stops the early, nearly instantaneous burst. In the polluted gas, density perturbations are allowed to grow via thermal instability (see also Fall \& Rees 1985), and radiative processes lead to a strong condensation in the cloud, which causes a burst of star formation, which in $\sim 10$ Myr produces a stellar population of $10^{5} \mathrm{M}_{\odot}$. The stellar mass profile calculated at $\sim 10 \mathrm{Myr}$ is characterized by a half-mass radius of $\sim 30 \mathrm{pc}$, that is, consistent with the values assumed in the AGB scenario.

The compact systems GC1 and ID11 present stellar densities, stellar masses and half-light radii consistent with the FG stellar masses and half-mass radii expected from the AGB scenario, and radii also compatible with the expectations of Nakasato et al. (2000).

It is worth stressing that the half-light radii are determined from 2D light distributions and should be regarded as underestimates of the 3D half-mass radii (typically of a factor of $\sim 3 / 4$ for a Sérsic profile), with a weak dependence on the Sérsic index; see Wolf et al. (2010). Our study shows the existence of very compact stellar objects in a redshift range in which GCs should be actively forming. Besides the sizes, in at least two cases (GC1 and ID11), also the ages of the stars are compatible with those expected for GCs caught during the formation of their stellar FG.

\subsection{The chance to observe a forming GC}

It may be useful to determine what is the probability to observe a GC in its forming phase (hereafter active GC). The physics of GC formation is quite uncertain (e.g. Renzini et al. 2015); in this work, we adopt a conservative approach by making plausible assumptions. To this aim, we consider two cases studied in the local Universe: the MW and the giant elliptical galaxy M87. This choice is due to the fact that MW-like mass star-forming galaxies might be rather common at high redshift and visible as Ly $\alpha$ emitters (see e.g. Dayal $\&$ Libeskind 2012). On the other hand, owing to its large stellar mass 
$\left(\sim 7 \times 10^{11} \mathrm{M}_{\odot} ;\right.$ Forte, Vega \& Faifer 2012) and to the presence of thousands of GCs (Bellini et al. 2015), M87 should be regarded as an extreme case and at high redshift it will be much rarer than MW-like mass galaxies.

It is known that the MW is surrounded by $N \simeq 150 \mathrm{GCs}$ (Harris 1996), whereas Bellini et al. (2015) have identified almost $N$ $\simeq 2000 \mathrm{GCs}$ in the core of M87. In our calculation, we assume that in a generic GC the first star formation event lasts $\Delta T_{\mathrm{GC}}=5 \mathrm{Myr}$. This time-scale corresponds to the typical age of local young massive clusters, which are known to be able to retain their gas for only a few Myr after the formation of their stars (e.g. Bastian et al. 2013), either because of a particularly strong feedback favouring gas expulsion, or because at least all the strongly gravitationally bound gas is converted into stars (Charbonnel et al. 2014).

The second assumption is that a GC formed sometime within the first two Gyrs after the big bang, corresponding to $z>3$ or $\Delta T_{\text {epoch }}$ $\simeq 2 \mathrm{Gyr}$.

We make the further conservative assumption that the formation epoch follows a flat distribution. Clearly, the assumption of a formation epoch peaking at some particular cosmic time would increase significantly the probability of catching an active GC around that cosmic time.

As we also ignore the original spatial distribution of proto-GCs, we assume that all GCs formed in the vicinity of the dark matter (DM) halo hosting the main galaxy, such that a typical observed field of view probes a sufficiently large volume to spatially include all the proto-GCs. In other words, we neglect if a GC has been acquired or formed 'in situ' (Katz \& Ricotti 2014). This assumption is corroborated by cosmological simulations, indicating that protoGCs form within a distance of tens of kpc proper from the main DM halo in which they are embedded, a volume well sampled by the observations (e.g. Kravtsov \& Gnedin 2005; Ricotti et al. 2016).

Under these simple hypotheses, the probability $(P)$ to observe at a given cosmic time (at $z>3$ ), $K$ (or $\geq K$ ) active GCs in a sample of $N(>K)$ GCs is

$P(K)=\left(\begin{array}{l}N \\ K\end{array}\right) p^{K}(1-p)^{N-K} ; \quad P(\geq K)=\sum_{i=k}^{N} P(i)$,

where the probability $p$ of the single case is $p=\Delta T_{\mathrm{GC}} / \Delta T_{\text {epoch }}=$ 0.0025 (as mentioned above by assuming a flat distribution). The probability that at least $1(5)$ out of $N$ GCs is caught during their formation is $\sim 30(56)$ per cent in the case of MW(M87), sometime at $z>3$. Clearly, these probabilities increase (decreases) if $\Delta T_{\mathrm{GC}}$ decreases (increases) or $N$ increases (decreases). It is worth stressing that if a non-flat formation history was assumed for proto-GCs, then we would have a specific cosmic epoch in which the frequency of simultaneously active GCs would be particularly high. In this respect, the probabilities computed with the assumptions described above should be regarded as conservative.

If we perform the same exercise by assuming a sample of $M$ galaxies, the probability will obviously increase. The probability that an event (with probability $P(K)$ ) occurs in at least one of the observed galaxies is $1-[1-P(K)]^{M}$.

The probabilities that 1,2 or $3 \mathrm{GCs}$ are active in an MW-like galaxy are $P(K)=25.8,4.8$ and 0.6 per cent, respectively. Moreover, the probabilities to observe 1, 2 or 3 active GCs in at least 1 out of 10 $(M=10)$ MW-like galaxies at $z>3$ are 84.6, 16.4 and 1.2 per cent. In the case of M87-like galaxies, these numbers increase significantly, although such massive objects are rarer. Fig. 8 shows the probability $P(K)$ as a function of $K$ calculated for various values of the sample size $M$ under the aforementioned hypotheses. In the same figure, the

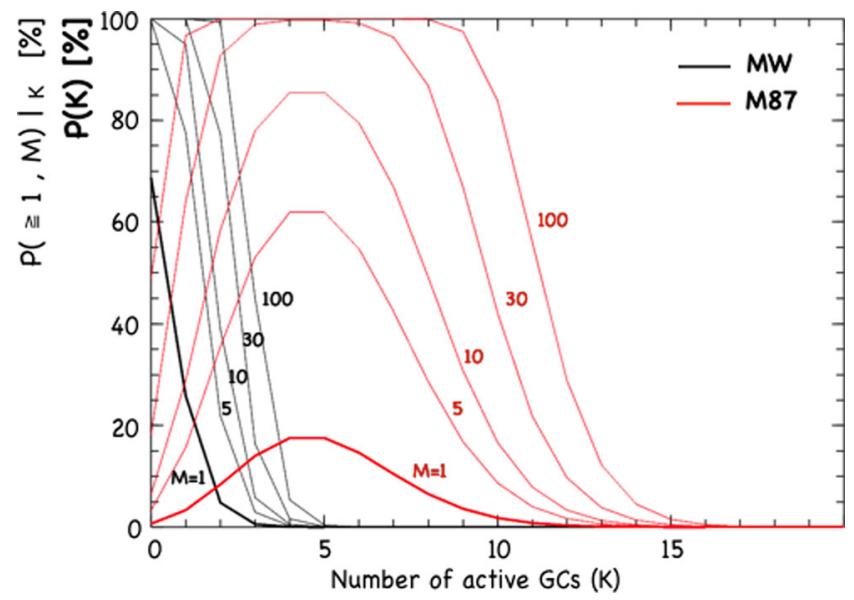

Figure 8. Two plots are shown in the same panel: the thick label in the $Y$-axis corresponds to the thick lines and the thin label to the thin curves. In particular, the thick black and red lines represent the probability $P(K)$ that $K$ GCs are simultaneously active (i.e. in a star-forming phase) at $z>3$ in an MW-like and an M87-like galaxy, respectively. For example, the probability $P(K)$ that 1(5) GCs are forming in the MW-like or M87-like galaxy is 26 and 18 per cent, respectively. The thin black and red lines are instead the probability $\left.(P \geq 1, M)\right|_{K}$ that $K$ simultaneously active GCs are present in at least one galaxy in a sample of $M$ MW-like and M87-like galaxies, respectively. The quantity $\left.(P \geq 1, M)\right|_{K}$ is computed for $M=1,5,10,30$ and 100. For example, the probability that $5(=K)$ GCs are active in one galaxy out of a sample of $10(=M)$ observed M87-like galaxies $\left.(P \geq 1, M)\right|_{K}$ is 85 per cent. By definition $\int_{0}^{\infty} P(K) \mathrm{d} K=1$.

black and red thick lines show the probabilities $P(K)$ as calculated from equation (3) for MW and M87, respectively, whose underlying area is one by definition. The thin lines show the probability that in at least one galaxy out of $M$ there are $K$ active GCs, for a given $P(K)\left(P \geq 1,\left.M\right|_{K}\right)$.

At the current stage, it is very difficult to compare the quantities shown in Fig. 8 with any of the observable properties discussed in the current work. To perform such a task, calculations of simultaneously active GCs per unit volume would be required, where number densities need to be extracted from cosmologically based models computing detailed merging trees, as performed, for example, in Ricotti (2002). The calculation described in this section shows that current surveys of lensed fields likely contain many active GCs and that the probability to observe a few of them simultaneously in a star-forming, active phase is quite high. A more detailed comparison between expected frequency and observed number of star-forming proto-GCs is postponed to the future, when larger samples of similar objects will become available. In the next section, the observability of such faint objects is discussed.

\subsection{Can we observe active GCs?}

Despite the current GC formation scenarios being still uncertain (e.g. Renzini et al. 2015), we report in Table 1 the possible ranges of a few relevant quantities expected during the formation of the FG stars in GCs of two different masses and within the AGB scenario (D'Ercole et al. 2008, 2016). D'Ercole et al. (2008) study the formation of one proto-GC of stellar mass $10^{6} \mathrm{M}_{\odot}$ and half-mass radius of $16 \mathrm{pc}$ and another more massive one, characterized by a stellar mass of $10^{7} \mathrm{M}_{\odot}$ and a half-mass radius of $35 \mathrm{pc}$. They assume an FG of stars already in place and focus mostly on the star formation history of SG stars. Assuming for the FG a constant SFR occurring on a time-scale of $5 \mathrm{Myr}$ as adopted in Section 4.2, we 
obtain for the low-mass and high-mass GC SFR values of 0.2 and $2 \mathrm{M}_{\odot} \mathrm{yr}^{-1}$, respectively. The two systems present stellar mass surface densities of 800 and $1700 \mathrm{M}_{\odot} \mathrm{pc}^{-2}$ for the less and the more massive cases, respectively. By means of the STARBURST99 models (Leitherer et al. 2014), we have calculated the expected monochromatic luminosity at $1500 \AA$ in the case of an instantaneous burst for the two stellar masses reported above and at an age of $\sim 5 \mathrm{Myr}$ after the burst. The result depends weakly on the adopted IMF and metallicity. The expected apparent magnitudes at a $1500 \AA$ rest frame are 30.5 and $\simeq 28$ at $z>3$ for the less massive and more massive clusters, respectively. This corresponds to absolute magnitudes fainter than $M_{\mathrm{UV}}=-17$ (consistently with estimates by Schaerer \& Charbonnel 2011). Clearly these values are elusive in the deepest non-lensed fields, such as the HUDF (Beckwith et al. 2006), particularly at magnitudes fainter than 29.5. However, as demonstrated in the previous sections, observations at the HUDF depth in regions of the sky magnified by lensing galaxy clusters (HFFs) have allowed us to probe extremely compact stellar systems $(<100 \mathrm{pc})$ at $z \simeq$ 3-6.

In addition, the expected Ly $\alpha$ line flux of proto-GCs is consistent with the observed values. The Ly $\alpha$ flux emerging from a protoGC can be estimated by assuming the case B recombination and the SFRs reported in Table 1, yielding 0.5-3 $\times 10^{-19} \mathrm{erg} \mathrm{s}^{-1} \mathrm{~cm}^{-2}$ (Schaerer 2003). Ly $\alpha$ line emission at these flux levels is in principle accessible in the deepest fields observed with MUSE (e.g. HDF-S; Bacon et al. 2015, see also fig. 6 in Vanzella et al. 2017). However, the detection of the continuum at magnitudes fainter than 30 is out of reach or at the very limit $(1 \sigma-2 \sigma$ detection) of the current deepest field surveys. This work demonstrates that strong gravitational lensing in deep fields allows us to overcome these limitations.

Finally, a previous study presenting considerable analogies with the current one and which is worth to mention is described in Ellis et al. (2001). Also in that case, a blind spectroscopic mode search was performed of objects lying nearby the critical line of the lensing cluster Abell 2218. A multiply lensed, intrinsically faint $(I \sim 30)$, compact $(<150 \mathrm{pc})$ system was found at $z \sim 5.6$ and with a very small stellar mass $\left(\sim 10^{6} \mathrm{M}_{\odot}\right)$. A young age for such a system ( $<2 \mathrm{Myr}$ ) was inferred from an upper limit on the stellar continuum, with the SFR deduced from its $\operatorname{Ly} \alpha$ emission. As the pioneering work of Ellis et al. (2001) shows several parallels with the one described here, it can be regarded as a remarkable forerunner of our results.

\subsection{Extremely faint, compact and dense forming objects}

The most compact objects, ID11 and GC1, show physical properties that are not far from those expected for proto-GCs. Before discussing this topic, we briefly report on their environment.

Object ID11 lives in a group of star-forming galaxies lying at the same redshift as confirmed by MUSE (Karman et al. 2016), including a Ly $\alpha$ nebula described by Caminha et al. (2016b) located at $\simeq 100 \mathrm{kpc}$ from ID11. Similarly, GC1 is part of a system including D1 and D2 at the same redshift, $z=6.145$. Also, system 1 in MACS J0416 (at $z=3.235$ ) is possibly part of a group of galaxies at similar redshift $(\Delta z<0.05)$, though in the current MUSE data no other galaxies have been confirmed at the same redshift (Caminha et al. 2016c). Therefore, our young, compact and low-mass objects are possibly sharing the same environment of (slightly) more massive and older systems. It is not clear if GC1 and ID11 will eventually merge with other systems or remain bound after the gas is removed (i.e. maintain their identity) until the present days. This is reminiscent of the present-day old dwarfs and GCs (older than
$10 \mathrm{Gyr}$ ) surrounding the local galaxies (including the MW) and implying that their ancestors were rapidly forming stars on a short time-scale (as we are observing here). Our objects might be the youngest counterparts of the extremely old systems observed today.

It is worth stressing that the intrinsic (delensed) magnitudes of the object discussed in this work span the range 29.0-31.4. In particular, GC1 with its intrinsic magnitude of 31.4 is a clear example of what is currently missed in the deepest surveys in the field, such as the HUDF (whose magnitude limit at $1 \sigma$ is $\simeq 30.5$ ). In this respect, the sources reported here open a new window to a very low mass/luminosity regime that unavoidably will need JWST and subsequently the extremely large telescopes for the characterization of their physical properties, especially for what concerns the absorption line science.

\subsubsection{Dynamical mass of ID11}

It is worth noting that ID11, the object with the best photometric measurements $(\mathrm{S} / \mathrm{N}>30)$ and with optical rest-frame spectroscopy, shows extremely narrow emission lines, both the highionization ultraviolet lines (e.g. C IV $\lambda \lambda 1548,1550$, He II $\lambda 1640$, $\left.\mathrm{O}_{\mathrm{III}}\right] \lambda \lambda 1661,1666, \mathrm{C}$ III] $\left.\lambda \lambda 1907,1909\right)$ and the optical lines (e.g. [O III] $\lambda \lambda 4959,5007)$. In particular, the oxygen (optical) lines [O III] $\lambda \lambda 4959,5007$ are well detected $(\mathrm{S} / \mathrm{N}=12-33)$ and barely resolved in our near-infrared X-Shooter spectrum $(R \simeq 5000)$, implying a very low velocity dispersion $\sigma_{v} \lesssim 20 \mathrm{~km} \mathrm{~s}^{-1}$ (see Vanzella et al. 2016a), and also a low dynamical mass. Following Maseda et al. (2013), we determine the dynamical mass using the virial relation:

$M_{\mathrm{dyn}}=C \frac{R_{\mathrm{e}} \sigma_{\mathrm{v}}^{2}}{G}$,

where $R_{\mathrm{e}}$ is the effective radius (assumed here to be the virial radius) and $\sigma_{\mathrm{v}}$ is the velocity dispersion (e.g. Maseda et al. 2014; Rhoads et al. 2014).

In general, it is well known that the coefficient $C$ is weakly dependent on the density profile when the velocity dispersion is measured over large apertures (in principle, over all the), and independent of orbital anisotropy (see e.g. Ciotti 1991, 1994). In particular, for values of the Sérsic index of $n \simeq 4-5$ as found for this system, $C$ varies between 4.65 and 3.67 (Bertin, Ciotti \& Del Principe 2002).

As discussed in Maseda et al. (2013), there are several potential systematic effects that may affect these estimates, including the fact that the measured half-light radius is not necessarily equal to the virial radius and that the dynamical structure might be irregular and not virialized. ID11 does not show an irregular morphology (plausibly close to spherical) and is well fitted by a single component profile, at least along the tangential direction.

Assuming that the above relation applies to ID11 and adopting $C=4$ as for the Green Pea galaxies (Erb et al. 2014; Maseda et al. 2014), the comparison with the stellar mass gives $M_{\text {dyn }} / M^{\star} \simeq 1$. Given the uncertainties in the estimation of $R_{\mathrm{e}}$ and the stellar mass, the ratio is fully consistent with a value of $\sim 1$.

Currently, there is no evidence that local GCs possess DM haloes (Heggie \& Hut 1996, but see also Ibata et al. 2013). It is possible that GCs were originally embedded in DM haloes, which have then been stripped by the tidal field of the host galaxy (Mashchenko \& Sills 2005), or that DM may be still present in the outer regions of the clusters (Bekki \& Yong 2012; Ibata et al. 2013). Our results obtained for ID11 imply a dynamical mass dominated by the stellar mass. Clearly, our result does not exclude the presence of DM on larger scales. In the future, it will be crucial to extend the study 
described in this section to larger samples, possibly comparing the results obtained using different emission lines to estimate the velocity dispersion $\sigma_{\mathrm{v}}$.

\subsection{Low-mass objects as contributors to cosmic reionization}

\subsubsection{Low-mass object might have large LyC leakage: the need for rest-frame optical spectroscopy}

As recent hydrodynamical simulations of proto-GCs have shown (Calura et al. 2015), the feedback of the stellar winds and SNe belonging to the FG can produce large and elongated hot cavities along which their interstellar gas is able to escape. In principle, these channels may also represent viable escape routes for ionizing photons. However, in order to assess whether proto-GCs might be important reionizing sources, an estimate of the time span over which their ISM remains rarefied and ionized is required, as well as the covering factor of the hot cavities and its evolution with time. Currently, work is in progress to investigate these aspects.

On the observational side, the ionizing radiation emitted by faint objects as well as their opacity at the $\operatorname{LyC}(\lambda<912 \AA)$ can be investigated only by pushing observations towards lower and lower luminosity regimes. While at relatively bright luminosities $\left(L>0.5 L^{\star}\right)$, the escape of ionizing radiation is not favoured, at least as far as observations at $z<4$ indicate (e.g. Izotov et al. 2016; Shapley et al. 2016; Vanzella et al. 2016b), and in general very modest (e.g. $<1-10$ per cent; Bridge et al. 2010; Vanzella et al. 2010, 2012b; Siana et al. 2010, 2015; Grazian et al. 2016; Guaita et al. 2016), in the low-luminosity domain it is still poorly explored.

Strong lensing magnification has allowed us to detect very faint sources (delensed $m_{1500} \gtrsim 29-30$ ) observed at $m_{1500} \gtrsim 26-27$ (implying magnification factors higher than 15). In order to possibly detect the LyC emerging from the same objects, we would need observations $\gtrsim 3$ mag deeper at $900 \AA$ than at $1500 \AA$ to probe an escaping radiation of 20 per cent, that is, magnitudes of the order of 29-30 in the LyC are still needed even in strongly lensed fields (e.g. Vanzella et al. 2012a).

In this challenging scenario, and since during the reionization epoch $(z>6)$, the LyC is not detectable owing to cosmic opacity, we must rely on indirect probes of LyC leakage, possibly calibrated on reference samples of LyC sources at $z<4$, when the IGM still allows us to directly detect the ionizing radiation. In this regard, recent progress has been made in the field of photoionization modelling (Inoue 2011; Jaskot \& Oey 2013; Nakajima \& Ouchi 2014; Zackrisson et al. 2017), subsequently confirmed by observations (e.g. de Barros et al. 2016; Vanzella et al. 2016b; Verhamme et al. 2017). In particular, line ratios in the rest-frame optical band like the O32 index $([\mathrm{O} \mathrm{III]}] \lambda 5007 /[\mathrm{O}$ II $] \lambda 3727)$ and specific properties of the $\mathrm{Ly} \alpha$ profile (Behrens, Dijkstra \& Niemeyer 2014; Verhamme et al. 2015; Dijkstra, Gronke \& Venkatesan 2016) can provide valuable indirect probes of the physical state of the interstellar medium and of the column density of neutral gas (e.g. density bounded or ionization bounded), as well as the connection with the ultraviolet spectral slope, the Balmer emission lines and the LyC leakage (Zackrisson, Inoue \& Jensen 2013; Zackrisson et al. 2017).

In Karman et al. (2016), a few lensed sources were already identified presenting a low column density of neutral gas $\left(<10^{18} \mathrm{~cm}^{-2}\right)$, possibly compatible with an LyC leakage (but still not confirmed directly). In particular, in the case of ID11, the VLT/X-Shooter near-infrared spectroscopy will reveal remarkable properties never observed before in such a faint and distant object (see Vanzella et al. 2016a). ID11 is a young, low-metallicity and dust-free object possibly captured during its first burst of star formation and confined in a small volume, surrounded by a shell of expanding gas. It also shows a low column density of neutral gas $\left(<10^{18.5} \mathrm{~cm}^{-2}\right.$, though not necessarily optically thin to $\mathrm{LyC}$ ) and a large $\mathrm{O} 32$ index (>10) compatible with a density-bounded condition in the ISM.

Therefore, rest-frame optical spectroscopy is crucial in this line of research. VLT/X-Shooter observations for system 1 at $z=3.235$ are ongoing (P.I. Vanzella) and will provide unique information at rest-frame optical wavelengths and at twice the spectral resolution of MUSE (as we did for ID11). It will also be essential to improve the dynamical mass estimate.

At $z=6.145$, the access to rest-frame optical nebular lines like [O II] $\lambda \lambda 3727,3729, \mathrm{H} \beta,[\mathrm{O}$ III] $\lambda \lambda 4959,5007$ and $\mathrm{H} \alpha$ (just to mention the most relevant ones) requires the NIRSpec instrument on JWST, as well as NIRCam for optical rest-frame morphology at $3-5 \mu \mathrm{m}$. The comparison of the ISM properties derived using the same spectral diagnostics (i.e. at the same rest-frame wavelengths) for these low-mass and extremely young star-forming objects will be crucial, especially in cases where a leakage of ionizing radiation is confirmed for sources at $z<4$. This will eventually represent a unique training set for the identification of the sources responsible for the reionization of the Universe.

\subsubsection{Lya nebulae as possible indirect signature of multiple proto GCs' ionization power}

Using the (Schaerer 2002, 2003) stellar population models and assuming a constant SFR of $1 \mathrm{M}_{\odot} \mathrm{yr}^{-1}$, after $\sim 3$ Myr the expected Ly $\alpha$ luminosity is $10^{42} \mathrm{erg} \mathrm{s}^{-1}$. If we rescale this value to our SFRs $\left(0.1 \mathrm{M}_{\odot} \mathrm{yr}^{-1}\right)$, we obtain an Ly $\alpha$ luminosity of the order of $10^{41} \mathrm{erg} \mathrm{s}^{-1}$. As discussed earlier in Section 4.2, the probability that at least 10 proto-GCs are active at the same time in M87-like galaxies observed at $z>3$ is not negligible ( $>10$ per cent). Therefore, assuming 10 proto-GCs simultaneously in place and emitting Ly $\alpha$ photons, their contribution to a diffuse Ly $\alpha$ emission might be relevant. In addition, assuming in each one of them an escape fraction of ionizing radiation higher than zero (e.g. > 30 per cent; Howard, Pudritz \& Klessen 2017), they could contribute to induce fluorescence in the surrounding medium, generating diffuse Ly $\alpha$ nebulae. Clearly, this cannot be regarded as the only mechanism responsible for the Ly $\alpha$ nebulae detected so far. However, a significant contribution from proto-GCs or dwarf galaxies cannot be excluded, especially if their stellar emission is not detectable even in the deepest field surveys (such as our GC1 source with magnitude 31.4). For instance, the origin of some of the Ly $\alpha$ nebulae discussed in Vanzella et al. (2017) is not well identified. Interestingly enough, a dozen proto GCs in action associated with a few high- $z$ galaxies can produce $\simeq 20$ per cent of the total Ly $\alpha$ luminosity observed in that case. Similarly, the clustering of faint objects around the main galaxy might also increase the Ly $\alpha$ visibility during the reionization epoch (Castellano et al. 2016b).

Are some of the Ly $\alpha$ nebulae observed so far an indirect signature of the integrated contribution from elusive proto-GCs? Again, JWST can investigate this issue by providing deeper images than what are available now, possibly revealing a multitude of currently undetected objects.

\section{CONCLUSIONS}

Deep observations provided by the HFF and deep VLT/MUSE integral field spectroscopy, coupled with high-precision lens models (based on tens of spectroscopic multiply imaged systems at 
$3<z<6$ ), have allowed us to identify extremely faint objects within the first two billion years after the big bang, in a still unexplored region of stellar mass and luminosity domains. These new regimes of mass and luminosity are relevant for our understanding of the physics of dwarf and GC formation at high redshift, as well as for the identification of sources possibly dominating the ionizing background (e.g. Wise et al. 2014; Yue et al. 2014).

The main results are the following.

(i) By taking advantage of (1) MUSE deep spectroscopy, (2) a detailed analysis of lensing magnification maps, (3) the spatial shapes of these selected sources (validated with lensing simulations) and (4) exploiting the Astrodeep HST photometry and SED fitting that includes nebular emission, we studied the faintest and most compact stellar systems at redshifts $\sim 3$ and $\sim 6$ currently known $\left(-14>M_{\mathrm{UV}}>-17\right)$, which are characterized by stellar masses in the range $10^{6}<M<20 \times 10^{6} \mathrm{M}_{\odot}$ and effective radii spanning the interval $\simeq 16-150 \mathrm{pc}$.

(ii) Two of our sources, GC1 and ID11, show stellar masses and SFR densities consistent with the values expected in multipopulation formation scenarios for GCs. In particular, ID11 also shows a dynamical mass (derived from optical rest-frame spectroscopy) similar to the stellar mass, suggesting a negligible DM content in this system. In addition, the detection of high-ionization lines like $\mathrm{C}_{\text {IV }} \lambda \lambda 1548,1550$ and $\mathrm{He}$ II $\lambda 1640$ (with a velocity dispersion of $\sigma_{\mathrm{v}} \simeq 20 \mathrm{~km} \mathrm{~s}^{-1}$ ) suggests that hot stars are present, with an outflowing gas with velocity $\simeq 50 \mathrm{~km} \mathrm{~s}^{-1}$ measured from line velocity offsets (Vanzella et al. 2016a). The same object also shows a low column density of neutral gas, lower than $10^{18.5} \mathrm{~cm}^{-2}$ (see Vanzella et al. 2016a). The other compact and dense object discovered at $z=6.145, \mathrm{GC} 1$, is perhaps the most intriguing source among those presented in this work, with an effective radius of $\simeq 20 \mathrm{pc}$ and an intrinsic stellar mass of $2-4 \times 10^{6} \mathrm{M}_{\odot}$. Its properties are very close to those expected for a proto-GC.

(iii) We have also detected extremely faint knots in the system at redshift 6.145 , whose delensed magnitudes are fainter than 32 . They are among the faintest objects at $z \sim 6$ ever found in any strongly lensed field. The very nature of these extremely faint star-forming regions will be better assessed with JWST and the extremely large telescopes.

The determination of sizes and physical properties (such as the stellar mass) of a large number of systems at $4 \leq z \lesssim 8$ will be greatly improved by means of JWST observations that will perform rest-frame optical spectroscopy and imaging with the NIRSpec and NIRCam instruments, respectively. In addition, the rest-frame near-infrared wavelengths will be accessible with the JWST/MIRI camera. ${ }^{4}$ Following the study of ID11 at $z=3.1169$ by means of VLT/X-Shooter near-infrared observations (see Vanzella et al. 2016a), JWST spectroscopy will allow us to:

(1) investigate the nature of the ionizing source from optical oxygen and Balmer line ratios, in combination with ultraviolet features,

(2) investigate the status of the interstellar medium through line ratios in the optical and ultraviolet rest frame (e.g. by calculating the $\mathrm{O} 32$ index and looking for density and/or ionization-bounded signatures),

(3) look for the presence of outflows possibly from the nebular emission of high-ionization lines (as we first attempted for ID11 with the C IV $\lambda \lambda 1548,1550$ doublet),
(4) perform direct estimates of the ionizing production rate from the Balmer lines, as well as to investigate the escaping ionizing radiation through indirect diagnostics calibrated at lower redshift (Vanzella et al. 2016b), specifically proposed for the characteristics of JWST (e.g. Zackrisson et al. 2013, 2017). It is worth noting that the current identification of photometric signatures of such nebular lines imprinted in the Spitzer/IRAC bands is even more complicated by the fact that at $5.5 \lesssim z \lesssim 6.6$ both the $3.6 \mu \mathrm{m}$ and $4.5 \mu \mathrm{m}$ bands are polluted by oxygen and $\mathrm{H} \alpha$ lines, respectively, introducing a degeneracy that prevents any clear measure of their equivalent widths (Smit et al. 2015). JWST/NIRSpec spectroscopy will remove this degeneracy.

Moreover, rest-frame optical and near-infrared imaging with NIRCam and MIRI will provide accurate estimates of the stellar masses and sizes (now inferred from the ultraviolet light at $z \sim 6$ ). The system at $z \sim 6$ also represents an ideal target for integral filed spectroscopy with JWST. In particular, the $3 \operatorname{arcsec} \times 3 \operatorname{arcsec}$ field of view provided by NIRSpec-IFU will produce a cube of 900 spaxels, which contains GC1, D1 and \#22692 sources in a single shot, as well as the possible stellar stream connecting all these features, enabling kinematics studies in the optical rest frame by using prominent lines such as [O II] $\lambda \lambda 3727,3729,[\mathrm{O}$ III] $\lambda \lambda 4959,5007$, $\mathrm{H} \beta$ and $\mathrm{H} \alpha$.

However, the study of ultraviolet absorption lines will require a good detection of the continuum $(\mathrm{S} / \mathrm{N}>5)$, achievable (thanks to gravitational lensing) for objects brighter than 28 at a $1500 \AA$ rest frame and addressable with future extremely large telescopes (e.g. E-ELT).

\section{ACKNOWLEDGEMENTS}

The referee, Richard Ellis, is acknowledged for several constructive comments that significantly improved the presentation of the results. We thank Alvio Renzini, F. Ferraro and E. Dalessandro for several useful discussions. CG acknowledges support by VILLUM FONDEN Young Investigator Programme through grant no. 10123. $\mathrm{KC}$ acknowledges funding from the European Research Council through the award of the Consolidator Grant ID 681627-BUILDUP. This study is based on observations collected at the European Southern Observatory for Astronomical research in the Southern hemisphere under ESO programmes P095.A-0653, P094.A-0115 (B) and ID 094.A-0525(A). MM, AM and PR acknowledge the financial support from PRIN-INAF 2014 1.05.01.94.02. MM acknowledges support from the Italian Ministry of Foreign Affairs and International Cooperation, Directorate General for Country Promotion. We also thank Wouter Karman for enlightening discussions over the last years.

\section{REFERENCES}

Alavi A. et al., 2014, ApJ, 780, 143

Alavi A. et al., 2016, ApJ, 832, 56

Atek H. et al., 2015, ApJ, 814, 69

Bacon R. et al., 2010, in McLean I. S., Ramsay S. K., Takami H., eds, Proc. SPIEConf. Ser. Vol. 7735, Ground-based and Airborne Instrumentation for Astronomy III. SPIE, Bellingham, p. 773508

Bacon R. et al., 2015, A\&A, 575, 75

Bastian N., Cabrera-Ziri I., Davies B., Larsen S. S., 2013, MNRAS, 436, 2852

Beckwith S. V. W. et al., 2006, AJ, 132, 1729

Behrens C., Dijkstra M., Niemeyer J. C., 2014, A\&A, 563, A77

Bekki K., Yong D., 2012, MNRAS, 419, 2063

${ }^{4}$ https://jwst.stsci.edu/instrumentation 
Bellini A. et al., 2015, ApJ, 805, 178

Bertin G., Ciotti L., Del Principe M., 2002, A\&A, 386, 149

Bouwens R. J., Illingworth G. D., Oesch P. A., Caruana J., Holwerda B., Smit R., Wilkins S., 2015, ApJ, 811, 140

Bouwens R. J., Smit R., Labbé I., Franx M., Caruana J., Oesch P., Stefanon M., Rasappu N., 2016a, ApJ, 831, 176

Bouwens R. J., Illingworth G. D., Oesch P. A., Atek H., Lam D., Stefanon M., 2016b, preprint (arXiv:1608.00966)

Bouwens R. J., Oesch P. A., Illingworth G. D., Ellis R. S., Stefanon M., 2016c, preprint (arXiv:1610.00283)

Bragaglia A., Carretta E., Sollima A., Donati P., D’Orazi V., Gratton R. G., Lucatello S., Sneden C., 2015, A\&A, 583, A69

Bridge C. R. et al., 2010, ApJ, 720, 465

Bruzual G., Charlot S., 2003, MNRAS, 344, 1000

Calura F., Ciotti L., Nipoti C., 2014, MNRAS, 440, 3341

Calura F., Few C. G., Romano D., D’Ercole A., 2015, ApJ, 814, L14

Caminha G. B. et al., 2016a, A\&A, 587, A80

Caminha G. B. et al., 2016b, A\&A, 595, A100

Caminha G. B. et al., 2016c, preprint (arXiv:1607.03462)

Castellano M. et al., 2012, A\&A, 540, A39

Castellano M. et al., 2014, A\&A, 566, A19

Castellano M. et al., 2016a, A\&A, 590, A31

Castellano M. et al., 2016b, ApJ, 818, L3

Charbonnel C., Chantereau W., Krause M., Primas F., Wang Y., 2014, A\&A, 569, L6

Ciotti L., 1991, A\&A, 249, 99

Ciotti L., 1994, Celest. Mech. Dyn. Astron., 60, 401

Cottrell P. L., Da Costa G. S., 1981, ApJ, 245, L79

D'Antona F., Caloi V., 2004, ApJ, 611, 871

D'Antona F., Caloi V., 2008, MNRAS, 390, 693

D’Ercole A., Vesperini E., D'Antona F., McMillan S. L. W., Recchi S., 2008, MNRAS, 391, 825

D'Ercole A., D’Antona F., Vesperini E., 2016, MNRAS, 461, 4088

Dayal P., Libeskind N. I., 2012, MNRAS, 419, L9

de Barros S. et al., 2016, A\&A, 585, A51

de Mink S. E., Pols O. R., Langer N., Izzard R. G., 2009, A\&A, 507, L1

Decressin T., Meynet G., Charbonnel C., Prantzos N., Ekström S., 2007, A\&A, 464, 1029

Decressin T., Baumgardt H., Charbonnel C., Kroupa P., 2010, A\&A, 516, A73

Denissenkov P. A., Hartwick F. D. A., 2014, MNRAS, 437, L21

Dijkstra M., Gronke M., Venkatesan A., 2016, ApJ, 828, 71

Downing J. M. B., Sills A., 2007, ApJ, 662, 341

Ellis R., Santos M. R., Kneib J.-P., Kuijken K., 2001, ApJ, 560, L119

Erb D. K. et al., 2014, ApJ, 795, 33

Fall S. M., Rees M. J., 1985, ApJ, 298, 18

Ferrara A., Loeb A., 2013, MNRAS, 431, 2826

Finlator K. et al., 2017, MNRAS, 464, 1633

Forte J. C., Vega E. I., Faifer F., 2012, MNRAS, 421, 635

Gratton R., Sneden C., Carretta E., 2004, ARA\&A, 42, 385

Grazian A. et al., 2016, A\&A, 585, A48

Guaita L. et al., 2016, A\&A, 587, A133

Harris W. E., 1996, AJ, 112, 1487

Heggie D. C., Hut P., 1996, in Hut P., Makino J., eds, Proc. IAU Symp. 174, Dynamical Evolution of Star Clusters: Confrontation of Theory and Observations. Kluwer, Dordrecht, p. 303

Howard C. S., Pudritz R. E., Klessen R. S., 2017, ApJ, 834, 40

Ibata R., Nipoti C., Sollima A., Bellazzini M., Chapman S. C., Dalessandro E., 2013, MNRAS, 428, 3648

Inoue A. K., 2011, MNRAS, 415, 2920

Izotov Y. I., Schaerer D., Thuan T. X., Worseck G., Guseva N. G., Orlitová I., Verhamme A., 2016, MNRAS, 461, 3683

Jaskot A. E., Oey M. S., 2013, ApJ, 766, 91

Karman W. et al., 2015, A\&A, 574, A11

Karman W. et al., 2016, A\&A, preprint (arXiv:1606.01471)

Katz H., Ricotti M., 2014, MNRAS, 444, 2377

Kawamata R., Ishigaki M., Shimasaku K., Oguri M., Ouchi M., 2015, ApJ, 804,103
Kimm T., Cen R., 2014, ApJ, 788, 121

King I., 1962, AJ, 67, 471

Kneib J.-P., Natarajan P., 2011, A\&AR, 19, 47

Koekemoer A. M. et al., 2014, Am. Astron. Soc. Meeting Abstr., 223, 254.02

Krause M., Charbonnel C., Decressin T., Meynet G., Prantzos N., Diehl R., 2012, A\&A, 546, L5

Kravtsov A. V., Gnedin O. Y., 2005, ApJ, 623, 650

Kroupa P., 2001, MNRAS, 322, 231

Kruijssen J. M. D., 2015, MNRAS, 454, 1658

Lardo C., Bellazzini M., Pancino E., Carretta E., Bragaglia A., Dalessandro E., 2011, A\&A, 525, A114

Leitherer C., Ekström S., Meynet G., Schaerer D., Agienko K. B., Levesque E. M., 2014, ApJS, 212, 14

Livermore R. C. et al., 2015, MNRAS, 450, 1812

Livermore R. C., Finkelstein S. L., Lotz J. M., 2017, ApJ, 835, 113

Lotz J. et al., 2014, Am. Astron. Soc. Meeting Abstr., 223, 254.01

Lotz J. M. et al., 2016, preprint (arXiv:1605.06567)

Maseda M. V. et al., 2013, ApJ, 778, L22

Maseda M. V. et al., 2014, ApJ, 791, 17

Mashchenko S., Sills A., 2005, ApJ, 619, 243

Meneghetti M. et al., 2008, A\&A, 482, 403

Meneghetti M., Rasia E., Merten J., Bellagamba F., Ettori S., Mazzotta P., Dolag K., Marri S., 2010, A\&A, 514, A93

Meneghetti M. et al., 2016, preprint (arXiv:1606.04548)

Merlin E. et al., 2016, A\&A, 590, A30

Nakajima K., Ouchi M., 2014, MNRAS, 442, 900

Nakasato N., Mori M., Nomoto K., 2000, ApJ, 535, 776

Peng C. Y., Ho L. C., Impey C. D., Rix H.-W., 2002, AJ, 124, 266

Peng C. Y., Ho L. C., Impey C. D., Rix H.-W., 2010, AJ, 139, 2097

Piotto G. et al., 2007, ApJ, 661, L53

Prantzos N., Charbonnel C., 2006, A\&A, 458, 135

Renzini A. et al., 2015, MNRAS, 454, 4197

Rhoads J. E., Malhotra S., Richardson M. L. A., Finkelstein S. L., Fynbo J. P. U., McLinden E. M., Tilvi V. S., 2014, ApJ, 780, 20

Ricotti M., 2002, MNRAS, 336, L33

Ricotti M., Parry O. H., Gnedin N. Y., 2016, ApJ, 831, 204

Salpeter E. E., 1955, ApJ, 121, 161

Schaerer D., 2002, A\&A, 382, 28

Schaerer D., 2003, A\&A, 397, 527

Schaerer D., Charbonnel C., 2011, MNRAS, 413, 2297

Schaerer D., de Barros S., 2009, A\&A, 502, 423

Shapley A. E., Steidel C. C., Strom A. L., Bogosavljević M., Reddy N. A., Siana B., Mostardi R. E., Rudie G. C., 2016, ApJ, 826, L24

Siana B. et al., 2010, ApJ, 723, 241

Siana B. et al., 2015, ApJ, 804, 17

Smit R. et al., 2015, ApJ, 801, 122

Terlevich R. et al., 2016, A\&A, 592, L7

Trenti M., Padoan P., Jimenez R., 2015, ApJ, 808, L35

Treu T. et al., 2015, ApJ, 812, 114

Vanzella E. et al., 2010, ApJ, 725, 1011

Vanzella E. et al., 2012a, MNRAS, 424, L54

Vanzella E. et al., 2012b, ApJ, 751, 70

Vanzella E. et al., 2014, ApJ, 783, L12

Vanzella E. et al., 2015, A\&A, 576, A116

Vanzella E. et al., 2016a, ApJ, 821, L27

Vanzella E. et al., 2016b, ApJ, 825, 41

Vanzella E. et al., 2017, MNRAS, 465, 3803

Verhamme A., Orlitová I., Schaerer D., Hayes M., 2015, A\&A, 578, A7

Verhamme A., Orlitova I., Schaerer D., Izotov Y., Worseck G., Thuan T. X., Guseva N., 2017, A\&A, 597, A13

Vesperini E., Heggie D. C., 1997, MNRAS, 289, 898

Wise J. H., Demchenko V. G., Halicek M. T., Norman M. L., Turk M. J., Abel T., Smith B. D., 2014, MNRAS, 442, 2560

Wolf J., Martinez G. D., Bullock J. S., Kaplinghat M., Geha M., Muñoz R. R., Simon J. D., Avedo F. F., 2010, MNRAS, 406, 1220

Yue B., Ferrara A., Vanzella E., Salvaterra R., 2014, MNRAS, 443, L20 
Zackrisson E., Inoue A. K., Jensen H., 2013, ApJ, 777, 39

Zackrisson E. et al., 2017, ApJ, 836, 78

\section{APPENDIX A: SIMULATING ID11}

We have assessed the robustness and uncertainties of the structural parameters reported above by performing end-to-end image simulations with the software SKYLENS (Meneghetti et al. 2008, 2010). As outlined also by Meneghetti et al. (2016), this code can be used to simulate HST observations, including the lensing effects produced by matter distributions along the line of sight to distant sources. In the simulations presented here, we use our lens model of AS1063 to lens some template sources placed at the redshift and predicted position of ID11. In the following tests, we make the simplified assumption that the sources have circular shape.

We begin by performing simulations without including lensing effects. Since the light profile and the size of the sources are known, we can test if our method to measure these properties using the fitting software GALFIT is robust. We generate source templates assuming Sérsic profiles with an index in the range [0.5-10] and effective radius in the range [20-640] pc. ${ }^{5}$ We assume that the apparent magnitude of these sources in the $F 814 \mathrm{~W}$ band is $m_{A B}=22.7$, which is chosen to provide a high $\mathrm{S} / \mathrm{N}$. We use GALFIT to retrieve the structural properties of the sources from the simulated observations, finding that profiles, radii and magnitude are correctly recovered only in the case of sources with $R_{\mathrm{e}}=640,320$ and $160 \mathrm{pc}$, the latter radius corresponding to 0.7 pixels at the redshift of ID11. When a smaller $R_{\mathrm{e}}$ is used, the profile parameters are only marginally recovered, showing the limits of the PSF deconvolution implemented by GALFIT. This latter case $\left(R_{\mathrm{e}}<100 \mathrm{pc}\right)$ corresponds to $R_{\mathrm{e}}<0.5$ pixels.

\footnotetext{
${ }^{5}$ The following values have been used: $20,40,60,80,160,320,640 \mathrm{pc}$.
}

Peng et al. (2010) show that objects with $R_{\mathrm{e}}$ smaller than half a pixel are not spatially resolved and severe systematics errors dominate any fit. ${ }^{6}$

Then, we repeat the above experiment by simulating the lensing effects produced by AS1063. Fig. A1 compares the results of the simulations with the real images of ID11 observed in the $F 814 \mathrm{~W}$ band. While a Gaussian profile (Sérsic index $n=0.5$ ) is clearly inconsistent with the morphology of the images A and B of ID11 for any value of $R_{\mathrm{e}}$, source models assuming Sérsic profiles with larger $n$ reproduce remarkably well the observed distortions, both in terms of their amplitude (i.e. the magnification) and direction. In particular, the best agreement is found for sources with $n \sim 4-5$ and $R_{\mathrm{e}} \sim 40-60$ pc (see again Fig. A1).

GALFIT fitting is performed on all the simulated images, and the recovered parameters are compared to those of the input sources. In this specific case, the presence of two multiple images with very similar magnification (A and B) allows us to further check the variation in the GALFIT measurements. In the cases $n=4$ and effective radii $[20-80] \mathrm{pc}$, we recover all the input parameters reasonably well. For example, in the case of the smallest source, $R_{\mathrm{e}}=20 \mathrm{pc}$, the measured effective radius is $R_{\mathrm{e}}=1.28 \pm 0.25$ pixels (in the tangential direction); that corresponds to $R_{\mathrm{e}}=1.28 \times 0.03 \times 7650 / \mu_{\mathrm{T}}$ $=18.1 \mathrm{pc}$. Similarly, the measured structural properties are consistent with the input source models for the other values of $n$ and $R_{\mathrm{e}}$ (see the summary table in Fig. A1). We can therefore conclude that (1) simulations validate the adopted method to infer the half-light radii and (2) objects with effective radii as small as $20 \mathrm{pc}$ are recoverable, assuming that they are tangentially magnified consistently to images A and B of ID11.

\footnotetext{
${ }^{6}$ Clearly, this depends also on the S/N of the image to fit and the quality of the PSF used. We limit our analysis to our PSF and for a bright object with magnitude 22.7, implying that for fainter, not-resolved objects the systematic errors are even more severe.
} 


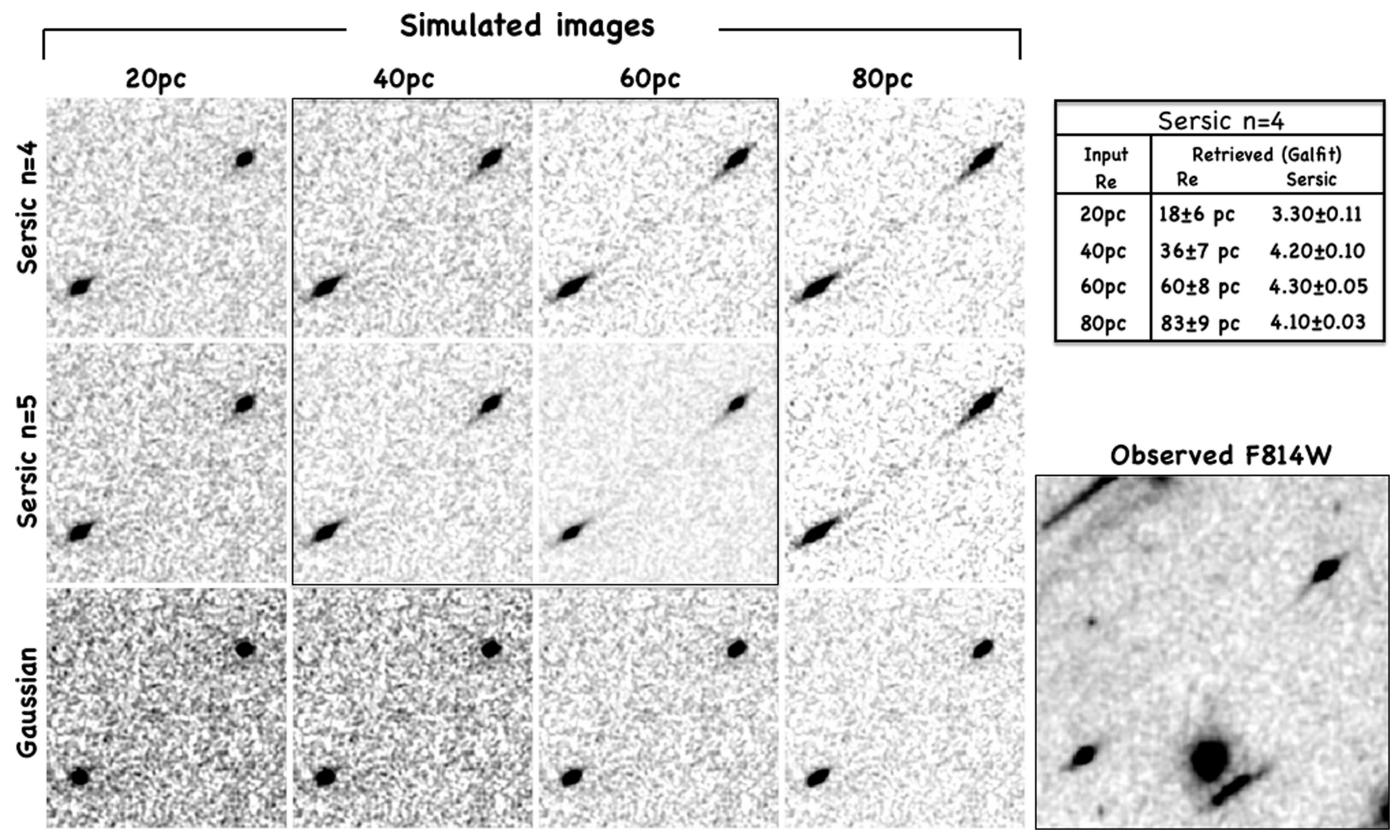

Figure A1. Simulated multiple images for ID11 are shown on the left-hand side $(4 \times 3$ panels) by adopting different light profiles (Sérsic, Gaussian) in the source plane and four effective radii $(20,40,60,80 \mathrm{pc})$. In the first two rows, a Sérsic index $n=4$ and 5 are adopted, while in the bottom row a two-dimensional Gaussian is assumed. On the right-hand side, the $F 814 \mathrm{~W}$ band image is shown. All the images have been smoothed with a Gaussian filter with a radius of 3 pixels to highlight the tails of the elongated images. Sérsic indexes $n \simeq 4-5$ and $R_{\mathrm{e}} \simeq 40-50 \mathrm{pc}$ well reproduce the data (see text for details). The top-right table compares the input radii and Sérsic index (only the $n=4$ case shown) of simulated images in the source plane with those retrieved by performing GALFIT fitting on the lensed simulated images.

This paper has been typeset from a $\mathrm{T}_{\mathrm{E}} \mathrm{X} / \mathrm{LAT} \mathrm{X}$ file prepared by the author. 